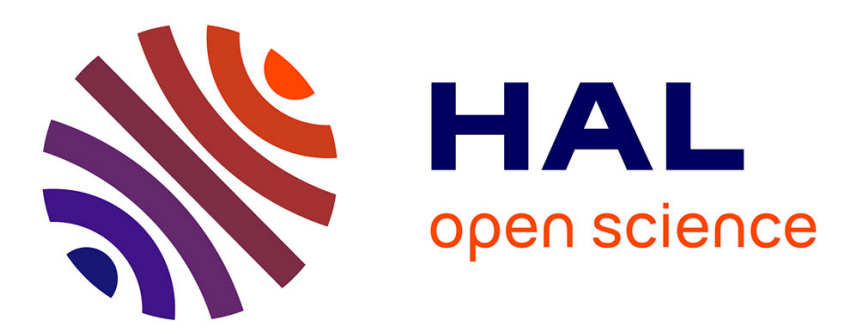

\title{
Spindle assembly in egg extracts of the Marsabit clawed frog, Xenopus borealis
}

Maiko Kitaoka, Rebecca Heald, Romain Gibeaux

\section{To cite this version:}

Maiko Kitaoka, Rebecca Heald, Romain Gibeaux. Spindle assembly in egg extracts of the Marsabit clawed frog, Xenopus borealis. Cytoskeleton, 2018, 75 (6), pp.244-257. 10.1002/cm.21444 . hal01863216v2

HAL Id: hal-01863216

https://hal-univ-rennes1.archives-ouvertes.fr/hal-01863216v2

Submitted on 14 Sep 2018

HAL is a multi-disciplinary open access archive for the deposit and dissemination of scientific research documents, whether they are published or not. The documents may come from teaching and research institutions in France or abroad, or from public or private research centers.
L'archive ouverte pluridisciplinaire HAL, est destinée au dépôt et à la diffusion de documents scientifiques de niveau recherche, publiés ou non, émanant des établissements d'enseignement et de recherche français ou étrangers, des laboratoires publics ou privés. 


\section{Spindle assembly in egg extracts of the Marsabit}

\section{clawed frog, Xenopus borealis}

Maiko Kitaoka $^{1}$, Rebecca Heald ${ }^{1 *(1)}$ and Romain Gibeaux ${ }^{1,2 *}$

${ }^{1}$ Department of Molecular and Cell Biology, University of California, Berkeley, CA 94720, USA

${ }^{2}$ Current address: Univ Rennes, CNRS, IGDR (Institute of Genetics and Development of Rennes) - UMR 6290, F-35000 Rennes, France

* Corresponding authors: romain.gibeaux@univ-rennes1.fr; bheald@, berkeley.edu

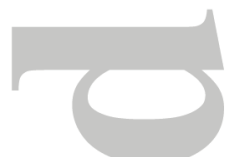

\section{RUNNING HEAD}

Xenopus borealis egg extract

KEYWORDS

Xenopus borealis, egg extracts, meiotic spindle, mitosis, TPX2, Ran

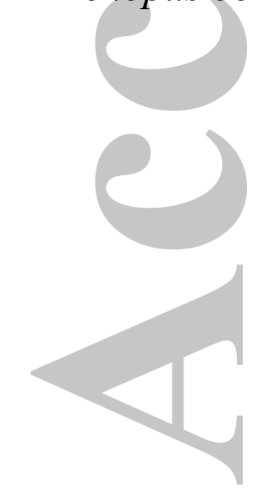




\begin{abstract}
Egg extracts of the African clawed frog Xenopus laevis have provided a cell-free system instrumental in elucidating events of the cell cycle, including mechanisms of spindle assembly. Comparison with extracts from the diploid Western clawed frog, Xenopus tropicalis, which is smaller at the organism, cellular and subcellular levels, has enabled the identification of spindle size scaling factors. We set out to characterize the Marsabit clawed frog, Xenopus borealis, which is intermediate in size between the two species, but more recently diverged in evolution from $X$. laevis than $X$. tropicalis. $X$. borealis eggs were slightly smaller than those of $X$. laevis, and slightly smaller spindles were assembled in egg extracts. Interestingly, microtubule distribution across the length of the $X$. borealis spindles differed from both $X$. laevis and $X$. tropicalis. Extract mixing experiments revealed common scaling phenomena among Xenopus species, while characterization of spindle factors katanin, TPX2, and Ran indicate that $X$. borealis spindles possess both $X$. laevis and $X$. tropicalis features. Thus, $X$. borealis egg extract provides a third in vitro system to investigate interspecies scaling and spindle morphometric variation.
\end{abstract}




\section{INTRODUCTION}

In all eukaryotes, cell division requires the spindle, a highly dynamic, self-organizing, microtubule-based apparatus that accurately segregates replicated chromosomes to daughter cells. Spindle microtubules assemble into a bipolar structure that attaches to chromosomes via their kinetochores and aligns them at the metaphase plate so that sister chromatids are oriented toward opposite spindle poles. Although the basic form and composition of the spindle is conserved, spindle structure adapts to changes in cell size and type, and varies dramatically both across and within species (Wühr et al., 2008; Crowder et al., 2015). While many of the molecules and mechanisms that orchestrate spindle assembly have been elucidated, it remains unclear how specific spindle architectures are established, and how this variation impacts spindle function.

Unfertilized eggs of the African clawed frog Xenopus laevis arrested in metaphase of meiosis II by cytostatic factor (CSF) (Masui and Markert, 1971) can be obtained in large quantities. Their fractionation by centrifugation yields a crude cytoplasmic extract that maintains the metaphase arrest and supports spindle assembly around sperm nuclei or chromatin-coated beads (Sawin and Mitchison, 1991; Heald et al., 1996). Remarkably, spindles formed in egg extracts of the smaller, related frog Xenopus tropicalis are shorter in length independent of DNA source, and mixing the two extracts produced spindles of intermediate size (Brown et al., 2007). It was found that compared to $X$. laevis, X. tropicalis possess smaller spindles due to (i) elevated activity of the hexameric AAA-ATPase microtubule severing enzyme katanin (Loughlin et al., 2010, 2011), and (ii) a higher concentration of the spindle assembly factor, TPX2 (targeting factor for Xklp2), which promotes association of the cross-linking spindle motor Eg5 and a shift of microtubule density from antiparallel overlap in the center of the spindle to the poles (Helmke and Heald, 2014). X. tropicalis spindles were also observed to possess distinct morphological features, including more robust kinetochore fibers and a more circular shape (Loughlin et al., 2011; Grenfell et al., 2016). Furthermore, whereas spindle assembly in X. laevis extracts required a chromatin-generated gradient of RanGTP (Kalab et al., 2002; Cavazza and Vernos, 2016), $X$. tropicalis spindle assembly was not affected by disruption of this pathway (Helmke and Heald, 2014). Therefore, not only spindle size, but also spindle architecture, morphology, and assembly mechanisms differ between these two species. 
To further address the conservation of spindle morphology, assembly, and scaling mechanisms across evolution, we decided to characterize a third Xenopus species, the Marsabit clawed frog Xenopus borealis. Whereas allotetraploid X. laevis (36 chromosomes) and diploid $X$. tropicalis (20 chromosomes) diverged 48 million years ago, $X$. borealis is more closely related to $X$. laevis, having diverged 17 million years ago (Session et al., 2016). Like $X$. laevis, X. borealis possesses an allotetraploid genome of 36 chromosomes. Interestingly, $X$. borealis was used extensively in the 1970 s to analyze the conservation of ribosomal nucleic acids compared to $X$. laevis (Brown et al., 1972; Brown and Sugimoto, 1974; Griswold et al., 1974; Leister and Dawid, 1975; Wellauer and Reeder, 1975; Ford and Brown, 1976) and was misidentified as Xenopus mulleri until 1977 (Brown et al., 1977). Revisiting this species and applying the egg extract methodology allowed us to further evaluate spindle morphometric variation and scaling in Xenopus. We show that $X$. borealis spindles are intermediate in size and display properties of both $X$. laevis and $X$. tropicalis, resulting in distinct features. These findings highlight the evolutionary plasticity of spindle morphology and assembly mechanisms, and introduce $X$. borealis as a third Xenopus species amenable to in vitro assays to investigate the molecular basis of spindle variation.

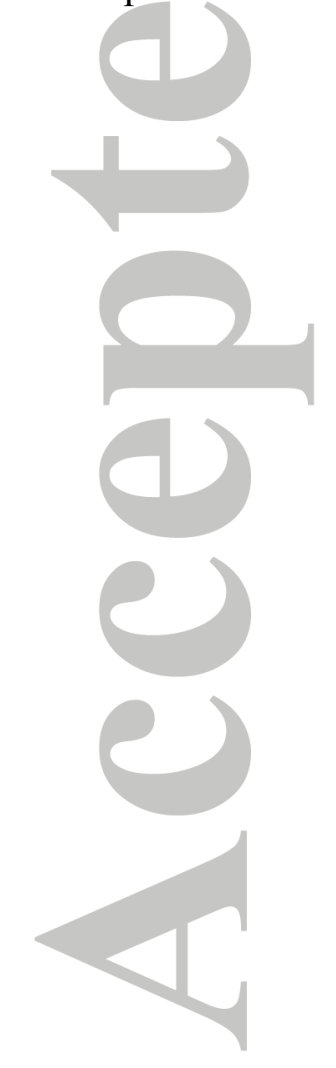




\section{RESULTS AND DISCUSSION}

In this study, we characterized $X$. borealis and spindle assembly in its egg extracts, using the two well-characterized Xenopus species, X. laevis and X. tropicalis, for comparison.

\section{$X$. borealis frogs, eggs, and sperm are morphologically distinct}

At $\sim 7 \mathrm{~cm}$ body length, adult $X$. borealis female frogs were intermediate in size between $X$. laevis $(\sim 10 \mathrm{~cm})$ and $X$. tropicalis $(\sim 4 \mathrm{~cm})$ and also displayed distinct physical traits including googly eyes and a pear-like body shape (Figure 1A). Measuring the size of $X$. borealis gametes revealed that $X$. borealis eggs averaged $1.2 \pm 0.02 \mathrm{~mm}$ in diameter, slightly smaller than $X$. laevis $(1.3 \pm$ $0.05 \mathrm{~mm})$ and larger than $X$. tropicalis $(0.75 \pm 0.02 \mathrm{~mm})$ (Figure 1B). Interestingly, $X$. borealis sperm cells were significantly longer (average $22 \pm 2.8 \mu \mathrm{m})$ than those of both X. laevis $(19 \pm 2.3$ $\mu \mathrm{m})$ and $X$. tropicalis $(13.5 \pm 2.1 \mu \mathrm{m})$ (Figure 1C), and similar size differences were observed comparing isolated sperm nuclei (Supplementary Figure S1) (Grainger, 2012). In vitro fertilization and live imaging revealed that the rate of early development of $X$. borealis and $X$. laevis embryos were similar (Figure 1D and Video 1) (Nieuwkoop and Faber, 1994). Nuclei isolated from stage $8\left(5 \mathrm{hpf}\right.$ ) embryos of $X$. borealis (average $2214 \pm 1016 \mu^{2}$ ) were comparable in size to those from $X$. laevis $\left(2010 \pm 1081 \mu \mathrm{m}^{2}\right)$, while $X$. tropicalis nuclei $(405 \pm$ $116 \mu^{2}$ ) were significantly smaller (Figure 1E). In summary, although $X$. borealis display distinct traits, our measurements show that like $X$. laevis and $X$. tropicalis, $X$. borealis egg size scales with adult animal size, and that $X$. borealis development and nuclear size is very similar to that of $X$. laevis. These findings are consistent with the well-characterized phenomenon that genome size scales with nuclear and cell size among amphibians (Levy and Heald, 2016).

\section{$X$. borealis egg extract recapitulates cell cycle events}

To evaluate the utility of $X$. borealis eggs for in vitro assays, we adapted published ovulation procedures to reproducibly obtain metaphase II, CSF-arrested eggs in sufficient amounts to prepare egg extracts (see Material and Methods). $X$. borealis eggs were dejellied and fractionated using standard protocols (Hannak and Heald, 2006; Maresca and Heald, 2006; Brown et al., 2007), and produced similar yields of cytoplasmic extract compared to X. laevis despite their smaller body and egg size. $X$. borealis egg extract was then combined with $X$. 
borealis sperm nuclei and assayed for spindle assembly. When added directly to CSF extract, sperm nuclei induced the formation of asters $\left(t=5^{\prime}\right)$, followed by half-spindles $\left(t=15^{\prime}\right)$ and bipolar CSF spindles $(\mathrm{t}=30$ - 45') (Figure 2A, B). Addition of calcium together with sperm nuclei induced exit from metaphase arrest and formation of interphase nuclei within $45 \mathrm{~min}$. Following the addition of fresh CSF extract, bipolar "cycled" spindles assembled from 75 min to 90 min (Figure 2A, C). We noted that $X$. borealis extracts formed spindles more quickly than $X$. laevis, almost as rapidly as $X$. tropicalis (unpublished data). These experiments show that, as for the other Xenopus species, $X$. borealis egg extracts are fully capable of recapitulating the cell cycle and assembling spindles in vitro.

\section{Xenopus species possess distinct spindle morphometrics}

We next examined some parameters of $X$. borealis spindle size and microtubule organization and compared them to in vivo meiosis II spindles as well as to spindles assembled in $X$. laevis and $X$. tropicalis egg extracts. $X$. borealis spindles assembled in vitro were similar in length from poleto-pole and width at the metaphase plate to meiotic spindles imaged in metaphase II-arrested eggs (length/width average $43.9 \pm 5.5 \mu \mathrm{m} / 21.7 \pm 3.6 \mu \mathrm{m}$ in vivo vs. $44.4 \pm 5.5 \mu \mathrm{m} / 23.4 \pm 5.9 \mu \mathrm{m}$ in extract) (Figure 3A). $X$. borealis extract was also capable of inducing bipolar spindle assembly around stage $8 X$. borealis embryo nuclei and $10 \mu \mathrm{m}$ diameter chromatin-coated beads (Halpin et al., 2011). Embryo nuclei spindles were slightly smaller than those formed around sperm nuclei (average length $39.7 \pm 4.4 \mu \mathrm{m}$, width $25.1 \pm 4.7 \mu \mathrm{m}$ ) (Figure 3B), while spindles assembled around DNA-coated beads were considerably shorter (average 22.4 $\pm 3.9 \mu \mathrm{m}$ ) (Figure 3C). These measurements demonstrate that like $X$. laevis and $X$. tropicalis, spindles formed in $X$. borealis egg extract mimic the size and morphology of the meiotic egg spindle, and that spindle assembly in vitro occurs in the presence or absence of centrosomes.

Despite their smaller egg size, we found that cycled $X$. borealis spindles were similar in length to $X$. laevis (average $41.9 \pm 4.1 \mu \mathrm{m}$ vs. $41.6 \pm 6.0 \mu \mathrm{m}$ ), but significantly narrower (average $19.7 \pm 3.7 \mu \mathrm{m}$ vs. $22.4 \pm 3.6 \mu \mathrm{m}$ ), thereby reducing spindle area and therefore total microtubule content. $X$. borealis spindles were both longer and wider than $X$. tropicalis spindles (average 23.2 $\pm 3.0 \mu \mathrm{m}$ in length, $10.4 \pm 2.6 \mu \mathrm{m}$ in width) (Figure 4A). Thus, $X$. borealis spindles scale with egg size and are intermediate in size between those of $X$. laevis and $X$. tropicalis. Interestingly, the three species each displayed unique spindle microtubule distributions across the spindle. As 
previously reported, $X$. laevis spindles showed a consistent plateau of tubulin intensity along the length of the spindle indicating the presence of an overlapping, tiled array of microtubules (Loughlin et al., 2010). In contrast, X. tropicalis tubulin intensity was greater at the poles and reduced in the center of the spindle at the metaphase plate (Helmke and Heald, 2014). Interestingly, $X$. borealis spindles displayed a similar increase in microtubule density at the poles as $X$. tropicalis, a similar density at the metaphase plate as $X$. laevis, and a unique dip in tubulin intensity between the poles and metaphase plate (Figure 4B). While similar microtubule distribution patterns were observed in CSF spindles, they were less distinct than in cycled spindles, potentially due to the absence of sister kinetochores and associated microtubules (Grenfell et al., 2016). X. borealis CSF spindles were also longer and wider than both $X$. laevis and $X$. tropicalis (Supplementary Figure S2). A difference in spindle morphology between spindles with replicated (cycled) and unreplicated (CSF) chromosomes has been observed previously and emphasizes the fact that spindle morphology can vary in different cellular contexts (Grenfell et al., 2016; Levy and Heald, 2016). We decided to focus on cycled spindle assembly for subsequent experiments, as these form around duplicated chromosomes with assembled kinetochores and thus are more physiologically relevant. Therefore, $X$. borealis fits into the Xenopus interspecies scaling regime, as the animals, eggs and meiotic spindles are all intermediate in size between the other two species. However, X. borealis microtubule arrays share some similarity with both species, leading to a unique spindle architecture.

\section{$X$. borealis spindles are sensitive to perturbation of the RanGTP gradient}

To compare spindle assembly mechanisms among the three species, we examined the role of the RanGTP gradient, which acts to release nuclear localization signal (NLS)-containing spindle assembly factors from importins near mitotic chromatin where the guanine nucleotide exchange factor RCC1 (Regulator of Chromosome Condensation 1) is localized, thereby promoting microtubule nucleation, stabilization, and crosslinking (Cavazza and Vernos, 2016). Perturbing the RanGTP gradient by the addition of Ran mutants, such as a dominant negative (T24N) or constitutively active, GTP-bound (Q69L) form, is detrimental to X. laevis spindle assembly, abolishing it completely or increasing ectopic microtubule nucleation and formation of multipolar spindles, respectively (Kalab et al., 1999, 2002). In contrast, we showed previously that $X$. tropicalis spindles are much less affected by perturbation of the RanGTP gradient 
(Helmke and Heald, 2014). To test the role of this pathway in X. borealis, we added the Ran mutants at the onset of spindle assembly. Upon addition of RanT24N, spindle assembly was strongly impaired, with dramatically reduced microtubule nucleation around sperm nuclei, while RanQ69L increased the number of multipolar spindle structures (Figure 5 and Supplementary Table 1). Thus, like $X$. laevis, $X$. borealis bipolar spindle assembly depends on the RanGTP gradient.

\section{Common spindle scaling mechanisms operate among Xenopus species}

It has been shown that hybrid embryos can be generated by fertilization of $X$. borealis eggs with sperm from either X. laevis and X. tropicalis (De Robertis and Black, 1979; Gibeaux et al., 2018), indicating compatibility between the $X$. borealis cytoplasm and the chromosomes of both species. Addition of either $X$. laevis or $X$. tropicalis sperm nuclei to $X$. borealis extract did not alter spindle microtubule distribution (Supplementary Figure S3A), consistent with previous studies indicating that egg cytoplasm composition is the primary determinant of spindle morphology (Brown et al., 2007). To determine whether maternal cytoplasmic factors affect spindle size similarly among the three Xenopus species, we mixed $X$. borealis egg extract with either X. laevis or X. tropicalis extract in the presence of $X$. laevis or X. tropicalis sperm nuclei, respectively. Whereas titration of $X$. borealis extract with $X$. laevis did not affect spindle dimensions (Figure 6A), $X$. tropicalis extract caused $X$. borealis spindles to shrink proportionately with the amount added (Figure 6B). Analysis of spindle microtubule distributions in mixed extracts revealed that these were also affected proportionately, favoring the tubulin distribution of the more abundant extract (Supplementary Figure S3B and C). These results are consistent with cytoplasmic mixing of $X$. laevis with $X$. tropicalis extracts, which also caused spindle shrinkage in a dose-dependent manner (Brown et al., 2007). Altogether, these data indicate that common scaling and microtubule organization mechanisms operate among the three Xenopus species.

\section{$X$. borealis spindles combine scaling factor features of both $X$. laevis and $X$. tropicalis}

To investigate the molecular basis of $X$. borealis spindle size and morphology, we compared the localization, abundance, and sequence of katanin and TPX2, two spindle scaling factors that contribute to spindle size differences between $X$. laevis and $X$. tropicalis, as well as the 
microtubule polymerase XMAP215, levels of which have been shown to regulate spindle length in Xenopus (Reber et al., 2013). XMAP215 levels and localization were similar for all three Xenopus species (Supplementary Figure S4). Katanin, a microtubule severing AAA-ATPase, has higher activity in $X$. tropicalis due to the absence of an inhibitory phosphorylation site (Serine 131) found in the $X$. laevis protein (Loughlin et al., 2011). Immunofluorescence and line scan analysis of spindles revealed that katanin localization on the $X$. borealis spindle displays a small but distinct increase at the metaphase plate as well as the spindle poles, while the localization on $X$. laevis spindles is more uniform and $X$. tropicalis shows katanin enrichment solely at the poles (Figure 7A). By Western blot, katanin levels did not differ significantly across the three species (Figure 7B). Although the amino acid sequence of $X$. borealis katanin is over $97 \%$ identical to that of both $X$. tropicalis and X. laevis, it contains the key inhibitory serine residue that regulates katanin activity in $X$. laevis (Supplementary Figure S5). It remains to be determined whether katanin activity is similarly regulated in X. borealis. TPX2 is a Ranregulated microtubule-associated protein that is found at three-fold higher concentrations in $X$. tropicalis compared to $X$. laevis. Increasing the level of TPX2 in $X$. laevis extracts to that of $X$. tropicalis was previously shown to reduce spindle length through its increased recruitment of the spindle motor Eg5 to spindle poles (Helmke and Heald, 2014). Interestingly, X. borealis spindles displayed an overall TPX2 localization more similar to $X$. tropicalis, with higher intensity at the spindle poles, but also possessed a central smaller peak of TPX2 reminiscent of $X$. laevis (Figure 7C). Levels of TPX2 in $X$. borealis egg extracts were two-fold higher than in $X$. laevis, but not elevated to $X$. tropicalis levels. (Figure 7D). Notably, the amino acid sequence of $X$. borealis TPX2 is more similar to $X$. laevis compared to $X$. tropicalis and contains the seven amino acid residues thought to reduce microtubule nucleation activity of $X$. laevis TPX2 (Supplementary Figure S6) (Helmke and Heald, 2014; Grenfell et al., 2016). Altogether, these results suggest that $X$. borealis spindle length results from its lower katanin activity similar to $X$. laevis, while its microtubule distribution is likely affected by increased levels of TPX2, which is more reminiscent of $X$. tropicalis.

Overall, these observations reveal that $X$. borealis meiotic spindles, although more similar in size and RanGTP-dependence to $X$. laevis, also possess some structural and molecular features characteristic of $X$. tropicalis. These findings support the idea that katanin and TPX2 are 
important spindle length control and assembly factors in Xenopus. Katanin-mediated microtubule severing activity has been shown to regulate spindle size in several organisms, including Caenorhabditis elegans, Xenopus, and human cells (McNally et al., 2006; Loughlin et al., 2011; Joly et al., 2016; Jiang et al., 2017), which is consistent with results from meiotic spindle assembly simulations showing that altering microtubule depolymerization rates robustly scales spindle length (Loughlin et al., 2010).

The role of TPX2 in spindle assembly and size control is more complicated. Depletion of the TPX2 ortholog D-TPX2 from Drosophila melanogaster syncytial embryos and S2 cells resulted in spindle shortening (Goshima, 2011; Hayward and Wakefield, 2014), and human cells require phosphorylation of TPX2 by Aurora A for normal spindle length (Bird and Hyman, 2008; Fu et al., 2015). In contrast, addition of recombinant TPX2 to X. laevis extracts decreased spindle length (Helmke and Heald, 2014), and we have observed similar effects on X. borealis spindles (unpublished data). Spindle-shrinking effects in Xenopus did not depend on Aurora A, but rather on a change in spindle microtubule distribution caused by increased recruitment of the cross-linking Eg5 motor to spindle poles (Helmke and Heald, 2014). TPX2 also regulates microtubule nucleation in the spindle (Petry et al., 2013; Scrofani et al., 2015; Petry, 2016; Alfaro-Aco et al., 2017). TPX2 has thus emerged as a central player of spindle assembly and organization in a wide variety of cell types (Ma et al., 2011; Helmke et al., 2013; Fu et al., 2015; Levy and Heald, 2016) and is overexpressed in many cancers (Chang et al., 2012; Neumayer et al., 2014). Our results highlight the importance of TPX2 levels and localization as determinants of meiotic spindle microtubule architecture. TPX2 localization (Figure 7C) correlates well with the distribution of tubulin intensity across all three species (Figure 4B), particularly at the spindle poles (Ma et al., 2011; Helmke and Heald, 2014). Despite having higher TPX2 levels, the greater sequence similarity of $X$. borealis TPX2 to the $X$. laevis protein (Supplementary Figure S6) may explain the increased sensitivity to Ran inhibition in $X$. borealis (Figure 5C), since a 7 amino acid sequence shown to decrease microtubule nucleation activity relative to $X$. tropicalis is present in both $X$. borealis and X. laevis TPX2 (Figure 7D) (Helmke and Heald, 2014). Altogether, our results provide clues to how TPX2 levels may tune meiotic spindle architecture and assembly pathways by altering microtubule nucleation and organization. 
In summary, Xenopus borealis provides a robust in vitro egg extract that adds to the characterized $X$. laevis and $X$. tropicalis systems, enabling further investigation of spindle assembly and interspecies scaling. Our findings highlight that egg meiosis II spindle architecture varies across Xenopus species and suggest that $X$. borealis spindles assemble with a combination of $X$. laevis as well as $X$. tropicalis features.

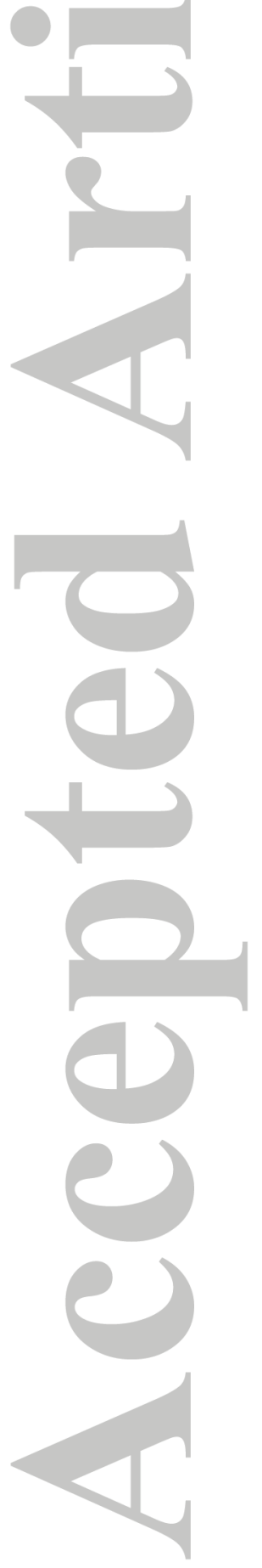




\section{MATERIALS AND METHODS}

\section{Chemicals}

Unless otherwise stated, all chemicals were purchased from Sigma-Aldrich, St. Louis, MO.

\section{Frog care}

All animal experimentation is this study was performed according to our Animal Use Protocol approved by the UC Berkeley Animal Care and Use Committee. Mature X. laevis, X. tropicalis, and $X$. borealis frogs were obtained from NASCO, WI. X. laevis, X. tropicalis, and X. borealis females were ovulated with no harm to the animals with a 6-, 3-, and 4-month rest interval, respectively. To obtain testes, males were euthanized by over-anesthesia through immersion in $\mathrm{ddH}_{2} \mathrm{O}$ containing $0.15 \%$ MS222 (Tricaine) neutralized with $5 \mathrm{mM}$ sodium bicarbonate prior to dissection, and then frozen at $-20^{\circ} \mathrm{C}$.

\section{Sperm nuclei purification}

$X$. laevis and $X$. tropicalis sperm nuclei were prepared and purified as previously described (Grainger, 2012). The following modifications were made for $X$. borealis sperm nuclei purification. $X$. borealis males were primed with $40 \mathrm{U}$ of PMSG 3 days prior to dissection and boosted with $100 \mathrm{U}$ hCG 12-24 h before dissection. Testes were removed and cleaned from all blood vessels and fat with forceps. Testes were then washed $3 \mathrm{X}$ in cold $1 \mathrm{X}$ MMR (1X MMR: $100 \mathrm{mM} \mathrm{NaCl}, 2 \mathrm{mM} \mathrm{KCl}, 2 \mathrm{mM} \mathrm{CaCl} 2,1 \mathrm{mM} \mathrm{MgCl}_{2}$ and $5 \mathrm{mM}$ HEPES-NaOH pH 7.6, 0.1 mM EDTA) and 3X in cold Nuclear Preparation Buffer (NPB) (2X NPB: $500 \mathrm{mM}$ sucrose, 30 mM HEPES, $1 \mathrm{mM}$ spermidine trihydrochloride, $0.4 \mathrm{mM}$ spermine tetrahydrochloride, $2 \mathrm{mM}$ dithiothreitol, $2 \mathrm{mM}$ EDTA) and placed in $1 \mathrm{~mL}$ of cold NPB for homogenization with scissors and a pestle. Samples were briefly spun to pellet the remaining tissue before transferring the supernatant to a new tube for centrifugation at $1500 \mathrm{~g}$ for $10 \mathrm{~min}$ at $4^{\circ} \mathrm{C}$ to pellet sperm. The pellet was resuspended in $1 \mathrm{~mL}$ NPB supplemented with $50 \mu \mathrm{L}$ of $10 \mathrm{mg} / \mathrm{mL}$ lysolecithin and incubated for $5 \mathrm{~min}$ at RT. The resuspended pellet was then added to $4 \mathrm{~mL}$ of cold NPB supplemented with $3 \% \mathrm{BSA}$ and spun at 3,000 RPM for 10 min at $4^{\circ} \mathrm{C}$. The resulting pellet was resuspended in $2 \mathrm{~mL}$ of cold NPB with $0.3 \%$ bovine serum albumin (BSA) and 30\% filtered glycerol. Finally, the pellet was resuspended in $500 \mu \mathrm{L}$ of cold NPB with $0.3 \%$ BSA and $30 \%$ 
filtered glycerol. Sperm nuclei were adjusted to a concentration of $10^{8}$ nuclei per $\mathrm{mL}$ using cold NPB with $0.3 \%$ BSA and $30 \%$ glycerol to make a $200 \mathrm{X}$ stock, aliquoted, frozen in liquid nitrogen, and stored at $-80^{\circ} \mathrm{C}$.

\section{In vitro fertilization}

$X$. borealis males were injected with $300 \mathrm{U}$ of human chorionic gonadotropin hormone (hCG) 12-24 $\mathrm{h}$ before dissection and testes were collected in Leibovitz L-15 Medium (Gibco - Thermo Fisher Scientific, Waltham, MA) supplemented with 10\% Fetal Bovine Serum (FBS; Gibco) for immediate use. $X$. laevis males were injected with $500 \mathrm{U}$ of hCG 12-24 h before dissection and testes were stored at $4^{\circ} \mathrm{C}$ in $1 \mathrm{X} \mathrm{MR}(100 \mathrm{mM} \mathrm{NaCl}, 1.8 \mathrm{mM} \mathrm{KCl}, 2 \mathrm{mM} \mathrm{CaCl} 2,1 \mathrm{mM} \mathrm{MgCl} 2$ and $5 \mathrm{mM}$ HEPES-NaOH $\mathrm{pH}$ 7.6) for 1-2 weeks.

$X$. borealis and $X$. laevis females were primed with $60 \mathrm{U}$ and $100 \mathrm{U}$, respectively, of pregnant mare serum gonadotrophin (PMSG, National Hormone and Peptide Program, Torrance, CA) at least $48 \mathrm{~h}$ before use and boosted with $300 \mathrm{U}$ and $500 \mathrm{U}$, respectively, of hCG 12-24 h before the experiment. $X$. borealis and $X$. laevis frogs were kept at $16^{\circ} \mathrm{C}$ in $0.5 \mathrm{X}$ and $1 \mathrm{X} \mathrm{MMR}$, respectively. $X$. borealis eggs were picked from the tub and deposited onto petri dishes coated with $1.5 \%$ agarose in $1 / 10 \mathrm{X}$ MMR. $X$. laevis females were squeezed gently to deposit eggs onto petri dishes coated with 1.5\% agarose in 1/10X MMR. Two X. borealis testes or $2 / 3$ of an $X$. laevis testis were collected and homogenized using scissors and a pestle in $1 \mathrm{~mL}$ of L-15 and $10 \%$ FBS. Any excess liquid in the petri dishes was removed, and the eggs were fertilized with $500 \mu \mathrm{L}$ of sperm solution per dish. Eggs were swirled in the solution to individualize eggs as much as possible and incubated for $5 \mathrm{~min}$. Dishes were flooded with $\mathrm{ddH}_{2} \mathrm{O}$, swirled and incubated for $10 \mathrm{~min}$, followed by buffer exchange to 1/10X MMR and incubation for $10 \mathrm{~min}$. The embryo jelly coats were then removed with a $3 \%$ cysteine solution in $\mathrm{ddH}_{2} \mathrm{O}-\mathrm{NaOH}, \mathrm{pH} 7.8$. After extensive washing ( $>4 \mathrm{X}$ ) with $1 / 10 \mathrm{X}$ MMR, embryos were incubated at $23^{\circ} \mathrm{C}$. At stage 23 , fertilized embryos were sorted and placed in fresh 1/10X MMR in new petri dishes coated with $1.5 \%$ agarose in $1 / 10 \mathrm{X}$ MMR.

All embryos were staged according to Nieuwkoop and Faber (Nieuwkoop and Faber, 1994).

\section{Embryo video imaging}


Imaging dishes were prepared using a homemade PDMS mold designed to print a pattern of 1 $\mathrm{mm}$ large wells in agarose that allowed us to image $4 X$. borealis or $X$. laevis embryos simultaneously within the $3 \mathrm{X} 4 \mathrm{~mm}$ camera field of view for each condition. Embryos were imaged from stage 2-3. Videos were taken simultaneously using two AmScope MD200 USB cameras (AmScope, Irvine, CA) each mounted on an AmScope SE305R stereoscope. Time lapse movies were acquired at a frequency of 1 frame every $10 \mathrm{~s}$ for $20 \mathrm{~h}$ and saved as Motion JPEG using a MATLAB (The MathWorks, Inc., Natick, MA) script. Movie post-processing (cropping, concatenation, resizing, addition of scale bar) was done using MATLAB and Fiji (Schindelin et al., 2012). All MATLAB scripts written for this study are available upon request. Two of the scripts used here were obtained through the MATLAB Central File Exchange: "videoMultiCrop" and "concatVideo2D" by Nikolay S.

\section{Embryo nuclei purification}

Following in vitro fertilization, $X$. borealis embryos were arrested at stage $8(\sim 5 \mathrm{hpf})$ in late interphase using $150 \mu \mathrm{g} / \mathrm{mL}$ cycloheximide in 1/10X MMR for $60 \mathrm{~min}$. Embryos were washed several times in Egg Lysis Buffer (ELB; $250 \mathrm{mM}$ sucrose, $50 \mathrm{mM} \mathrm{KCl,} 2.5 \mathrm{mM} \mathrm{MgCl}_{2}$, and 10 mM HEPES pH 7.8) supplemented with LPC (10 $\mu \mathrm{g} / \mathrm{mL}$ each leupeptin, pepstatin, chymostatin), cytochalasin D $(100 \mu \mathrm{g} / \mathrm{mL})$, and cycloheximide $(100 \mu \mathrm{g} / \mathrm{mL})$, packed in a tabletop centrifuge at $200 \mathrm{~g}$ for $1 \mathrm{~min}$, crushed with a pestle, and centrifuged at $10,000 \mathrm{~g}$ for $10 \mathrm{~min}$ at $16^{\circ} \mathrm{C}$. The cytoplasmic extract containing endogenous embryonic nuclei was collected, supplemented with $8 \%$ glycerol, aliquoted, frozen in liquid nitrogen, and stored at $-80^{\circ} \mathrm{C}$.

\section{$X$. borealis egg extract}

$X$. borealis metaphase-arrested egg extracts were prepared similarly to $X$. laevis and $X$. tropicalis (Maresca and Heald, 2006; Brown et al., 2007), with the following modifications. X. borealis female frogs were primed with $60 \mathrm{U}$ PMSG at least 48 hours before use and boosted with $300 \mathrm{U}$ hCG 12-24 hours before the experiment. Frogs were kept at $16^{\circ} \mathrm{C}$ in $0.5 \mathrm{X}$ MMR. The eggs were dejellied in a 3\% cysteine solution in $1 \mathrm{X}$ XB-salts (20X: $2 \mathrm{M} \mathrm{KCl}, 20 \mathrm{mM} \mathrm{MgCl} 2,2 \mathrm{mM} \mathrm{CaCl}$ ), pH 7.8, and washed in CSF-XB (100 mM KCl, $0.1 \mathrm{mM} \mathrm{CaCl}_{2}, 3 \mathrm{mM} \mathrm{MgCl}$, $50 \mathrm{mM}$ sucrose, 10 $\mathrm{mM}$ K-EGTA, $10 \mathrm{mM}$ K-HEPES, $\mathrm{pH}$ 7.8). CSF-XB buffer was exchanged for CSF-XB supplemented with LPC $(10 \mu \mathrm{g} / \mathrm{mL})(\mathrm{CSF}-\mathrm{XB}+) .1 \mathrm{~mL}$ of CSF-XB + supplemented with 
cytochalasin D $(100 \mu \mathrm{g} / \mathrm{mL})$ was added to the bottom of an ultracentrifuge tube (SW-55, Beckman) before loading eggs into the tube. Extract tubes were placed in adapter tubes and spun at $1600 \mathrm{~g}$ for $1 \mathrm{~min}$ to pack the eggs. The excess liquid was removed and the eggs crushed by centrifugation at $10,200 \mathrm{rpm}$ for $16 \mathrm{~min}$ at $16^{\circ} \mathrm{C}$. The cytoplasmic fraction was then removed and supplemented with LPC (1:1000 dilution; $10 \mathrm{mg} / \mathrm{mL}$ stock), cytochalasin D (1:500 dilution; 10 $\mathrm{mg} / \mathrm{mL}$ stock), energy (1:50 dilution; 50X stock: $190 \mathrm{mM}$ creatine phosphate, $25 \mathrm{mM}$ adenosine triphosphate, $25 \mathrm{mM} \mathrm{MgCl}$, $2.5 \mathrm{mM} \mathrm{K-EGTA} \mathrm{pH} \mathrm{7.7),} \mathrm{and} \mathrm{rhodamine-tubulin} \mathrm{(1:300} \mathrm{dilution;}$ $65.8 \mu \mathrm{g} / \mathrm{uL}$ stock) for spindle assembly reactions. Sperm nuclei, embryo nuclei, or $10 \mu \mathrm{m}$ chromatin-coated beads, prepared as previously described (Halpin et al., 2011), were used as a source of DNA for spindle assembly.

\section{$X$. laevis and $X$. tropicalis egg extracts and mixing experiments}

$X$. laevis and $X$. tropicalis metaphase arrested egg extracts were prepared and spindle reactions conducted as previously described (Maresca and Heald, 2006; Brown et al., 2007). X. laevis or $X$. tropicalis extracts were mixed in different proportions to $X$. borealis extract and supplemented with either $X$. laevis or $X$. tropicalis sperm nuclei, respectively.

\section{Extract treatments}

To examine sensitivity to perturbations of the RanGTP gradient, $X$. laevis, $X$. tropicalis, and $X$. borealis sperm nuclei were added to their respective egg extracts and cycled through interphase. Ran T24N (final concentration $1 \mu \mathrm{M}$ ) and Q69L (final concentration $5 \mu \mathrm{M}$ ) mutant proteins, purified as previously described (Helmke and Heald, 2014), were added to the interphase extract reaction when fresh CSF extract was added to induce the extract to cycle back to mitosis.

\section{Spindle spin-down and immunofluorescence}

Spindle reactions were prepared, spun-down, and processed for immunofluorescence as previously described (Hannak and Heald, 2006). Briefly, the extract reactions were fixed for 510 minutes with $2.5 \%$ formaldehyde and spun down at $10,200 \mathrm{rpm}$ for $16 \mathrm{~min}$ at $16^{\circ} \mathrm{C}$. The coverslips were incubated for $30 \mathrm{~s}$ in cold methanol, washed in PBS+NP40, and blocked overnight in PBS $+5 \%$ BSA at $4^{\circ} \mathrm{C}$. The anti-TPX2 (1:150 dilution, unpublished), anti-katanin p60 (1:500 dilution) (Loughlin et al., 2011), or anti-XMAP215 (1:200, Tony Hyman \& Kazu 
Kinoshita) rabbit antibodies were added for $1 \mathrm{~h}$ in PBS $+5 \%$ BSA. After washing with PBS+NP40, the coverslips were incubated with 1:1000 anti-rabbit antibody coupled to Alexa Fluor 488 (Invitrogen - Thermo Fisher Scientific, Waltham, MA) for 30 min and then with 1:1000 Hoechst for $5 \mathrm{~min}$. The coverslips were then washed and mounted for imaging with Vectashield (Vector Laboratories, Burlingame, CA).

\section{Egg whole mount immunofluorescence}

Metaphase-arrested $X$. borealis eggs were fixed for 1-3 $\mathrm{h}$ at RT using MAD fixative (2 parts of methanol (Thermo Fisher Scientific, Waltham, MA), 2 parts of acetone (Thermo Fisher Scientific, Waltham, MA), 1 part of DMSO), and stored overnight at $-20^{\circ} \mathrm{C}$ in fresh MAD fixative. Embryos were then processed as previously described (Lee et al., 2008) with some modifications. Following gradual rehydration in 0.5X SSC (1X SSC: $150 \mathrm{mM} \mathrm{NaCl}, 15 \mathrm{mM} \mathrm{Na}$ citrate, $\mathrm{pH} 7.0$ ), embryos were bleached with $2 \% \mathrm{H}_{2} \mathrm{O}_{2}$ (Thermo Fisher Scientific, Waltham, $\mathrm{MA}$ ) in $0.5 \mathrm{X}$ SSC containing 5\% formamide for 2-3 $\mathrm{h}$ under light, then washed in PBT, a PBS solution containing $0.1 \%$ Triton X-100 (Thermo Fisher Scientific, Waltham, MA) and $2 \mathrm{mg} / \mathrm{ml}$ BSA. Embryos were blocked in PBT supplemented with 10\% goat serum (Gibco - Thermo Fisher Scientific, Waltham, MA) and 5\% DMSO for 1-3 h and incubated overnight at $4^{\circ} \mathrm{C}$ in PBT supplemented with 10\% goat serum and the primary antibodies. We used the following antibodies: 1:500 mouse anti-beta tubulin (E7; Developmental Studies Hybridoma Bank, Iowa City, IA), 1:350 rabbit anti-histone H3 (ab1791; Abcam, Cambridge, MA). Embryos were then washed 4 X 2 h in PBT and incubated overnight in PBT supplemented with 1:500 goat antimouse secondary antibody coupled to Alexa Fluor 488 and 1:500 goat anti-rabbit secondary antibody coupled to Alexa Fluor 568 (Invitrogen - Thermo Fisher Scientific, Waltham, MA). Embryos were then washed 4 X $2 \mathrm{~h}$ in PBT and gradually dehydrated in methanol. Embryos were finally cleared in Murray's clearing medium (2 parts of Benzyl Benzoate, 1 part of Benzyl Alcohol). Embryos were placed in a chamber made using a flat nylon washer (Grainger, Lake Forest, IL) attached with nail polish (Sally Hansen, New York, NY) to a slide and covered by a coverslip, and filled with Murray’s clearing medium for confocal microscopy.

\section{Confocal microscopy}


Confocal microscopy was performed on an upright Zeiss LSM 780 NLO AxioExaminer using the Zeiss Zen software. Embryos were illuminated with an LED light source (X-cite 120LED). For the imaging of histone $\mathrm{H} 3$ and tubulin, embryos were imaged using a Plan-Apochromat $20 \mathrm{x} / 1.0$ Water objective and laser powers of $12 \%$, on multiple $1024 \times 1024$ px plans spaced of $0.683 \mu \mathrm{m}$ in $\mathrm{Z}$. Images are mean averages of 2 scans with a depth of 16 bits. Channels were acquired simultaneously, and pinhole size was always chosen to correspond to 1 airy unit.

\section{Imaging and quantification}

Eggs were imaged using the Leica Application Suite (v4.9; Leica Microsystems, Buffalo Grove, IL) with a Wild M7A stereoscope equipped with a Leica MC170HD camera. Sperm cells, sperm nuclei, embryo nuclei, and spindles were imaged using micromanager software (Edelstein et al., 2014) with an Olympus BX51 microscope equipped with an ORCA-ER camera (Hamamatsu Photonics, Hamamatsu city, Japan), and with an Olympus UPlan FL 40x/0.75 air objective. All measurements were made using Fiji. The spindle tubulin, katanin, TPX2, and XMAP215 intensity line scans were measured using an automated Java ImageJ plugin developed by Xiao Zhou (Heald lab, UC Berkeley; https://github.com/XiaoMutt/AiSpindle).

\section{Western blot and analysis}

$1 \mu \mathrm{L}$ of egg extract for 3 independent extracts for each species was subjected to SDS-PAGE (12\% gel for katanin, $8 \%$ gel for TPX2 and XMAP215) and transferred to a nitrocellulose membrane. The anti-TPX2 (1:500 dilution, unpublished), anti-katanin p60 (1:1000 dilution) (Loughlin et al., 2011), or anti-XMAP215 (1:1000, Tony Hyman \& Kazu Kinoshita) rabbit antibodies and anti-Ran (1:2000 dilution, BD Biosciences, San Jose, CA) mouse antibody were added for $1 \mathrm{~h}$ or overnight at $4^{\circ} \mathrm{C}$ in PBS $+0.1 \%$ Tween $-20+5 \%$ milk. Secondary antibodies (goat anti-rabbit IRDye 800 or goat anti-mouse IRDye 680) were added at 1:10,000 for $1 \mathrm{~h}$ in PBS $+0.1 \%$ Tween-20. Blots were scanned with an Odyssey Infrared Imaging System (LI-COR Biosciences), and band intensity was quantified with Fiji.

\section{Protein sequence alignments}


Multiple sequence alignments were performed using Clustal Omega (default parameters). Sequence similarities were determined by pairwise alignments using EMBOSS Needle (default parameters).
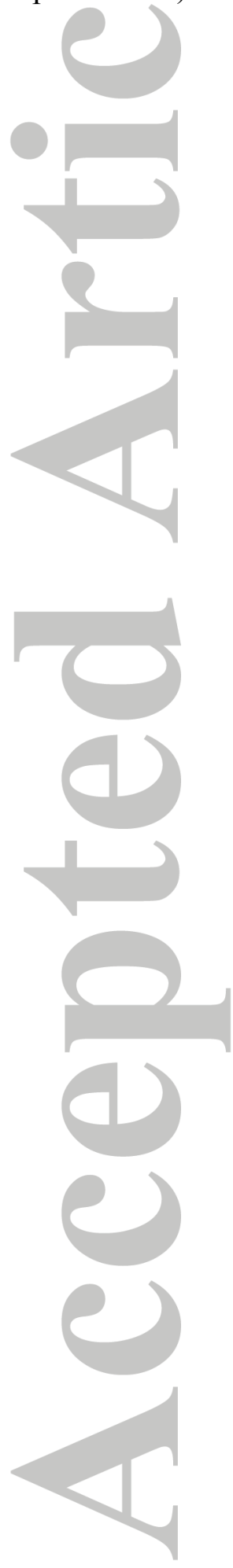


\section{ACKNOWLEDGEMENTS}

We thank members of the Heald lab for support and fruitful discussions, in particular Xiao Zhou for his line scan plugin and Ambika Nadkarni for the purification of Ran mutants. We thank Sofia Medina-Ruiz, Austin Mudd, and Dan Rokhsar for providing early access to X. borealis katanin, TPX2, and XMAP215 sequences. The confocal microscopy performed in this work was done at the UC Berkeley CRL Molecular Imaging Center, supported by NSF DBI-1041078. MK was supported by the UC Berkeley MCB department NIH training grant 4T32GM007232-40. RH was supported by NIH R35 GM118183 and the Flora Lamson Hewlett Chair. RG was supported by an HFSP long term fellowship LT 0004252014-L.

\section{AUTHOR CONTRIBUTIONS}

M.K. performed the experiments and analyzed the data, with help from R.G. M.K. prepared the figures and wrote the manuscript with input from R.H. and R.G.

\section{AUTHOR INFORMATION}

The authors declare no competing financial interests. Correspondence should be addressed to R.G. (romain.gibeaux@univ-rennes1.fr) and R.H. (bheald@berkeley.edu). 


\section{REFERENCES}

Alfaro-Aco, R., Thawani, A., and Petry, S. (2017). Structural analysis of the role of TPX2 in branching microtubule nucleation. J. Cell Biol. 216, 983-997.

Bird, A. W., and Hyman, A. A. (2008). Building a spindle of the correct length in human cells requires the interaction between TPX2 and Aurora A. J. Cell Biol. 182, 289-300.

Brown, D. D., Dawid, I. B., and Reeder, R. H. (1977). Xenopus borealis misidentified as Xenopus mulleri. Dev. Biol. 59, 266-267.

Brown, D. D., and Sugimoto, K. (1974). The structure and evolution of ribosomal and 5S DNAs in Xenopus laevis and Xenopus mulleri. Cold Spring Harb. Symp. Quant. Biol. 38, 501-505.

Brown, D. D., Wensink, P. C., and Jordan, E. (1972). A comparison of the ribosomal DNA's of Xenopus laevis and Xenopus mulleri: the evolution of tandem genes. J. Mol. Biol. 63, 57-73.

Brown, K. S., Blower, M. D., Maresca, T. J., Grammer, T. C., Harland, R. M., and Heald, R. (2007). Xenopus tropicalis egg extracts provide insight into scaling of the mitotic spindle. J. Cell Biol. 176, 765-770.

Cavazza, T., and Vernos, I. (2016). The RanGTP Pathway: From Nucleo-Cytoplasmic Transport to Spindle Assembly and Beyond. Front. Cell Dev. Biol. 3.

Chang, H., Wang, J., Tian, Y., Xu, J., Gou, X., and Cheng, J. (2012). The TPX2 gene is a promising diagnostic and therapeutic target for cervical cancer. Oncol. Rep. 27, 13531359.

Crowder, M. E., Strzelecka, M., Wilbur, J. D., Good, M. C., Von Dassow, G., and Heald, R. (2015). A comparative analysis of spindle morphometrics across metazoans. Curr. Biol. $25,1542-1550$.

Edelstein, A. D., Tsuchida, M. a, Amodaj, N., Pinkard, H., Vale, R. D., and Stuurman, N. (2014). Advanced methods of microscope control using $\mu$ Manager software. J. Biol. Methods 1, 10 .

Ford, P. J., and Brown, R. D. (1976). Sequences of 5S ribosomal RNA from Xenopus mulleri and the evolution of 5S gene-coding sequences. Cell 8, 485-493.

Fu, J., Bian, M., Xin, G., Deng, Z., Luo, J., Guo, X., Chen, H., Wang, Y., Jiang, Q., and 
Zhang, C. (2015). TPX2 phosphorylation maintains metaphase spindle length by regulating microtubule flux. J. Cell Biol. 210, 373-383.

Gibeaux, R. et al. (2018). Paternal chromosome loss and metabolic crisis contribute to hybrid inviability in Xenopus. Nature 553, 337-341.

Goshima, G. (2011). Identification of a TPX2-like microtubule-associated protein in Drosophila. PLoS One 6.

Grainger, R. M. (2012). Xenopus tropicalis as a Model Organism for Genetics and Genomics: Past, Present and Future. Methods Mol. Biol., 3-15.

Grenfell, A. W., Strzelecka, M., Crowder, M. E., Helmke, K. J., Schlaitz, A. L., and Heald, R. (2016). A versatile multivariate image analysis pipeline reveals features of Xenopus extract spindles. J. Cell Biol. 213, 127-136.

Griswold, M. D., Brown, R. D., and Tocchini-Valentini, G. P. (1974). An analysis of the degree of homology between 28S rRNA from Xenopus laevis and Xenopus mulleri. Biochem. Biophys. Res. Commun. 58, 1093-1103.

Halpin, D., Kalab, P., Wang, J., Weis, K., and Heald, R. (2011). Mitotic spindle assembly around RCC1-coated beads in Xenopus egg extracts. PLoS Biol. 9.

Hannak, E., and Heald, R. (2006). Investigating mitotic spindle assembly and function in vitro using Xenopus laevis egg extracts. Nat. Protoc. 1, 2305-2314.

Hayward, D., and Wakefield, J. G. (2014). Chromatin-mediated microtubule nucleation in Drosophila syncytial embryos. Commun. Integr. Biol. 7.

Heald, R., Tournebize, R., Blank, T., Sandaltzopoulos, R., Becker, P., Hyman, A., and Karsenti, E. (1996). Self-organization of microtubules into bipolar spindles around artificial chromosomes in Xenopus egg extracts. Nature 382, 420-425.

Helmke, K. J., and Heald, R. (2014). TPX2 levels modulate meiotic spindle size and architecture in Xenopus egg extracts. J. Cell Biol. 206, 385-393.

Helmke, K. J., Heald, R., and Wilbur, J. D. (2013). Interplay between spindle architecture and function, Elsevier Inc.

Jiang, K., Rezabkova, L., Hua, S., Liu, Q., Capitani, G., Altelaar, A. F. M., Heck, A. J. R., Kammerer, R. A., Steinmetz, M. O., and Akhmanova, A. (2017). Microtubule minus-end regulation at spindle poles by an ASPM-katanin complex. Nat. Cell Biol. 19, 480-492. Joly, N., Martino, L., Gigant, E., Dumont, J., and Pintard, L. (2016). Microtubule-severing 
activity of the AAA + ATPase Katanin is essential for female meiotic spindle assembly. Development 143, 3604-3614.

Kalab, P., Pu, R. T., and Dasso, M. (1999). The Ran GTPase regulates mitotic spindle assembly. Curr. Biol. 9, 481-484.

Kalab, P., Weis, K., and Heald, R. (2002). Visualization of a Ran-GTP gradient in interphase and mitotic Xenopus egg extracts. Science 295, 2452-2456.

Lee, C., Kieserman, E., Gray, R. S., Park, T. J., and Wallingford, J. (2008). Whole-Mount Fluorescence Immunocytochemistry on Xenopus Embryos. Cold Spring Harb. Protoc. 3, $1-5$.

Leister, D. E., and Dawid, I. B. (1975). Mitochondrial ribosomal proteins in Xenopus laevis/X. mulleri interspecific hybrids. J. Mol. Biol. 96, 119-123.

Levy, D. L., and Heald, R. (2016). Biological scaling problems and solutions in amphibians. Cold Spring Harb. Perspect. Biol. 8.

Loughlin, R., Heald, R., and Nédélec, F. (2010). A computational model predicts Xenopus meiotic spindle organization. J. Cell Biol. 191, 1239-1249.

Loughlin, R., Wilbur, J. D., McNally, F. J., Nédélec, F. J., and Heald, R. (2011). Katanin contributes to interspecies spindle length scaling in Xenopus. Cell 147, 1397-1407.

Ma, N., Titus, J., Gable, A., Ross, J. L., and Wadsworth, P. (2011). TPX2 regulates the localization and activity of Eg5 in the mammalian mitotic spindle. J. Cell Biol. 195, 8798.

Maresca, T. J., and Heald, R. (2006). Methods for studying spindle assembly and chromosome condensation in Xenopus egg extracts. Methods Mol. Biol. (Clifton, NJ) $322,459-474$.

Masui, Y., and Markert, C. L. (1971). Cytoplasmic control of nuclear behavior during meiotic maturation of frog oocytes. J. Exp. Zool., 129-146.

McNally, K., Audhya, A., Oegema, K., and McNally, F. J. (2006). Katanin controls mitotic and meiotic spindle length. J. Cell Biol. 175, 881-891.

Neumayer, G., Belzil, C., Gruss, O. J., and Nguyen, M. D. (2014). TPX2: Of spindle assembly, DNA damage response, and cancer. Cell. Mol. Life Sci. 71, 3027-3047.

Nieuwkoop, P. D., and Faber, J. (1994). Normal table of Xenopus laevis (Daudin), Garland Publishing. 
Petry, S. (2016). Mechanisms of Mitotic Spindle Assembly. Annu. Rev. Biochem. 85, 659683.

Petry, S., Groen, A. C., Ishihara, K., Mitchison, T. J., and Vale, R. D. (2013). Branching microtubule nucleation in Xenopus egg extracts mediated by augmin and TPX2. Cell 152, $768-777$.

Reber, S. B., Baumgart, J., Widlund, P. O., Pozniakovsky, A., Howard, J., Hyman, A. A., and Jülicher, F. (2013). XMAP215 activity sets spindle length by controlling the total mass of spindle microtubules. Nat. Cell Biol. 15, 1116-1122.

De Robertis, E. M., and Black, P. (1979). Hybrids of Xenopus laevis and Xenopus borealis express proteins from both parents. Dev. Biol. 68, 334-339.

Sawin, K. E., and Mitchison, T. J. (1991). Mitotic Spindle Assembly by Two Different Pathways in Vitro. J. Cell Biol. 112, 925-940.

Schindelin, J. et al. (2012). Fiji: an open-source platform for biological-image analysis. Nat. Methods 9, 676-682.

Scrofani, J., Sardon, T., Meunier, S., and Vernos, I. (2015). Microtubule nucleation in mitosis by a RanGTP-dependent protein complex. Curr. Biol. 25, 131-140.

Session, A. M. et al. (2016). Genome evolution in the allotetraploid frog Xenopus laevis. Nature 538, 336-343.

Wellauer, P. K., and Reeder, R. H. (1975). A comparison of the structural organization of amplified ribosomal DNA from Xenopus mulleri and Xenopus laevis. J. Mol. Biol. 94, $151-161$.

Wühr, M., Chen, Y., Dumont, S., Groen, A. C., Needleman, D. J., Salic, A., and Mitchison, T. J. (2008). Evidence for an Upper Limit to Mitotic Spindle Length. Curr. Biol. 18, $1256-1261$. 


\section{FIGURE LEGENDS}

\section{FIGURE 1: Characterization of the Marsabit clawed frog, $X$. borealis}

(A) Relative body size of $X$. borealis frogs compared to $X$. laevis and $X$. tropicalis. (B) Comparison and quantification of egg size. Egg size (diameter in $\mathrm{mm}$ ) is plotted as boxplots for $X$. laevis (green), X. tropicalis (blue), and X. borealis (purple). Each dot represents an individual $\operatorname{egg}(\mathrm{n}=89$ for $X$. laevis, $\mathrm{n}=132$ for $X$. tropicalis, and $\mathrm{n}=77$ for $X$. borealis, from 3 females for each species). Representative images of eggs for each species are shown (right). Scale bar is 200 $\mu \mathrm{m}$. (C) Comparison and quantification of sperm size. Sperm head length $(\mu \mathrm{m})$ is plotted as boxplots for $X$. laevis (green), X. tropicalis (blue), and $X$. borealis (purple). Each dot represents an individual sperm cell $(\mathrm{n}=288$ for $X$. laevis, $\mathrm{n}=278$ for $X$. tropicalis, and $\mathrm{n}=310$ for $X$. borealis, from 3 males for each species). Representative images of sperm cells for each species are shown (right). Scale bar is $20 \mu \mathrm{m}$. (D) Early embryo development at room temperature for $X$. laevis and $X$. borealis. Embryos were staged according to Nieuwkoop and Faber (Nieuwkoop and Faber, 1994). Data for X. laevis embryos $(\mathrm{n}=12)$ are blotted in green and X. borealis $(\mathrm{n}=$ 12) in purple. Error bars show the standard deviation. (E) Stage 8 embryo nuclei comparison and quantification. Representative images of nuclei isolated from stage 8 X. laevis, X. tropicalis, and $X$. borealis embryos are shown with DNA signal. Scale bar is $10 \mu \mathrm{m}$. Nuclear size (area in $\mu \mathrm{m}^{2}$ ) is plotted as boxplots for X. laevis (green), X. tropicalis (blue), and X. borealis (purple). Each dot represents an individual nucleus $(\mathrm{n}=68$ for $X$. laevis, $\mathrm{n}=71$ for $X$. tropicalis, and $\mathrm{n}=84$ for $X$. borealis). In $\mathbf{B}, \mathbf{C}$, and $\mathbf{E}$, the thicker black line indicates the average, and the outlined gray box represents the standard deviation. Statistical significance was determined by a two-tailed, twosample unequal variance t-test.

\section{FIGURE 2: $X$. borealis egg extracts at a glance}

(A) Schematic of extract preparation and spindle assembly reactions. (B) Images of spindle assembly intermediates in CSF extract reactions. (C) Images of spindle assembly intermediates in cycled extract reactions. Images in B-C of representative structures are shown with tubulin (red) and DNA (blue) signal. Scale bar is $20 \mu \mathrm{m}$.

\section{FIGURE 3: $X$. borealis CSF egg extracts reconstitute spindle assembly}


(A) In vivo $X$. borealis metaphase-arrested egg spindles compared to spindles assembled in CSF extract. Scale bar is $20 \mu \mathrm{m}$. Spindle length (left) and width (right) are plotted ( $\mu \mathrm{m})$ as columns for $X$. borealis spindles from in vivo metaphase-arrested eggs (light purple) and formed in in vitro egg extract (dark purple). Error bars represent the standard deviation. (B) Spindles assembled around stage $8 X$. borealis embryo nuclei added to $X$. borealis egg extract. Scale bar is $20 \mu \mathrm{m}$. Spindle length (left) and width (right) are plotted $(\mu \mathrm{m})$ as boxplots. Each dot represents an individual spindle measurement ( $\mathrm{n}=310$, from 3 independent egg extracts). (C) Spindles assembled around single $10 \mu \mathrm{m}$ chromatin-coated beads in $X$. borealis egg extract. Scale bar is $10 \mu \mathrm{m}$. Length $(\mu \mathrm{m})$ is plotted as a boxplot. Each dot represents an individual spindle measurement ( $\mathrm{n}=219$, from 4 independent egg extracts). Representative images in A-C are shown with tubulin (top) and DNA (middle) signals individually and merged (bottom). In B-C, the thicker black line indicates the average, and the outlined gray box represents the standard deviation.

\section{FIGURE 4: Analysis of $X$. borealis cycled spindles}

(A) Quantification of $X$. borealis cycled spindle dimensions. Length (left), width (middle), and area approximated as an ellipse (right) are plotted $\left(\mu \mathrm{m}\right.$ or $\left.\mu \mathrm{m}^{2}\right)$ as boxplots for $X$. laevis (green), $X$. tropicalis (blue), and $X$. borealis (purple) spindles. Each dot represents an individual spindle measurement $(\mathrm{n}=256$ spindles for $X$. laevis, $\mathrm{n}=241$ for $X$. tropicalis, and $\mathrm{n}=493$ for $X$. borealis, from 3 independent egg extracts for each species), the thicker black line indicates the average, and the outlined gray box represents the standard deviation. Statistical significance was determined by a two-tailed, two-sample unequal variance t-test. (B) Microtubule fluorescence intensity distribution in X. laevis (green), X. tropicalis (blue), and X. borealis (purple) spindles. Line scans of rhodamine-tubulin signal along the length of the spindle were measured ( $\mathrm{n}=257$ spindles for $X$. laevis, $\mathrm{n}=242$ for $X$. tropicalis, and $\mathrm{n}=470$ for $X$. borealis, from 3 independent egg extracts for each species). Spindle length was normalized to $100 \%$ and tubulin intensities were normalized within each dataset. Average tubulin intensities were plotted for each species' spindles. Error bars represent the standard deviation. Representative images of spindles formed from $X$. laevis, $X$. tropicalis, and $X$. borealis sperm nuclei in their respective egg extracts are shown with tubulin signal individually (left) and merged with DNA signal (right). Scale bar is 20 $\mu \mathrm{m}$. 


\section{FIGURE 5: Sensitivity of $X$. borealis spindles to perturbation of the RanGTP gradient}

(A) Phenotype categories quantified in Xenopus egg extract spindle assembly reactions containing Ran mutants. Representative images in $X$. laevis extracts are shown with tubulin (red) and DNA (blue) signals. Scale bar is $20 \mu \mathrm{m}$. Bar graphs of the percentage of spindles in each category are shown for (B) untreated extracts, (C) RanT24N treated extracts, and (D) RanQ69L treated in Xenopus extracts. In B-D, percentages of bipolar (dark gray), multipolar (polka dots), and no spindles found (light gray) are plotted for $X$. laevis, $X$. tropicalis, and $X$. borealis extracts (3 independent extracts were quantified for each species). Error bars represent the standard deviation. Statistical significance was determined by a two-tailed, 3x2 Fisher Exact test.

\section{FIGURE 6: Titration of $X$. borealis egg extract with other Xenopus egg extracts}

(A) $X$. borealis extract mixed with $X$. laevis extract. Spindle length (left panel) and width (right panel) are plotted as columns, with colors proportionate to the amount of $X$. laevis (green) added to $X$. borealis (purple) $(\mathrm{n}=341$ for $100 \%$ X. borealis, $0 \% X$. laevis; $\mathrm{n}=350$ for $75 \%$ X. borealis, $25 \% X$. laevis $; \mathrm{n}=331$ for $50 \% X$. borealis, $50 \% X$. laevis $; \mathrm{n}=313$ for $25 \%$ X. borealis, $75 \% X$. laevis; and $\mathrm{n}=267$ for $0 \% X$. borealis, $100 \%$ X. laevis over 3 independent experiments). (B) $X$. borealis extract mixed with $X$. tropicalis extract. Spindle length (left panel) and width (right panel) are plotted as columns, with colors proportionate to the amount of $X$. tropicalis (blue) added to $X$. borealis (purple) $(\mathrm{n}=295$ for $100 \%$ X. borealis, $0 \%$ X. tropicalis; $\mathrm{n}=306$ for $75 \%$ $X$. borealis, 25\% X. tropicalis; $\mathrm{n}=295$ for 50\% X. borealis, 50\% X. tropicalis; $\mathrm{n}=337$ for $25 \%$ X. borealis, $75 \% X$. tropicalis; and $\mathrm{n}=334$ for $0 \% X$. borealis, $100 \%$ X. tropicalis over 3 independent experiments). In A-B, spindle length and width were normalized to the length and width, respectively, of $100 \% X$. borealis CSF spindles. Error bars represent the standard deviation. Representative images are shown with tubulin (red) and DNA (blue) signal. Scale bar is $20 \mu \mathrm{m}$.

\section{FIGURE 7: Localization of scaling factors katanin and TPX2 on X. borealis spindles}

(A) Katanin immunofluorescence staining of X. laevis (green), X. tropicalis (blue), and $X$. borealis (purple) spindles. Line scans of Alexa Fluor 488 signal along the length of the spindle were measured $(\mathrm{n}=385$ spindles for $X$. laevis, $\mathrm{n}=408$ for $X$. tropicalis, and $\mathrm{n}=348$ for $X$. 
borealis, from 3 independent egg extracts for each species). (B) Western blots of $X$. laevis, $X$. tropicalis, and $X$. borealis extracts probed for katanin. (C) TPX2 immunofluorescence staining of $X$. laevis (green), X. tropicalis (blue), and X. borealis (purple) spindles. Line scans of Alexa Fluor 488 signal along the length of the spindle were measured $(\mathrm{n}=406$ spindles for $X$. laevis, $\mathrm{n}$ $=433$ for $X$. tropicalis, and $\mathrm{n}=418$ for $X$. borealis, from 3 independent egg extracts for each species). (D) Western blots of $X$. laevis, X. tropicalis, and X. borealis extracts, probed for TPX2. In $\mathbf{A}$ and $\mathbf{C}$, spindle length was normalized to $100 \%$ and katanin or TPX2 intensities, respectively, were normalized within each dataset. Average TPX2 intensities were plotted for each species' spindles. Error bars represent the standard deviation. Representative images of spindles formed from $X$. laevis, $X$. tropicalis, and $X$. borealis sperm nuclei in their respective egg extracts are shown with katanin or TPX2 grayscale signal (left) and merged with tubulin and DNA signals (right). Scale bar is $20 \mu \mathrm{m}$. In B and D, katanin or TPX2 band integrated densities are plotted as columns for X. laevis (green), X. tropicalis (blue), and X. borealis (purple) extracts (3 independent extracts are shown for each species). Band intensities were normalized to the integrated density of the corresponding Ran loading control. Error bars represent the standard deviation. Statistical significance was determined by a two-tailed, two-sample unequal variance t-test.

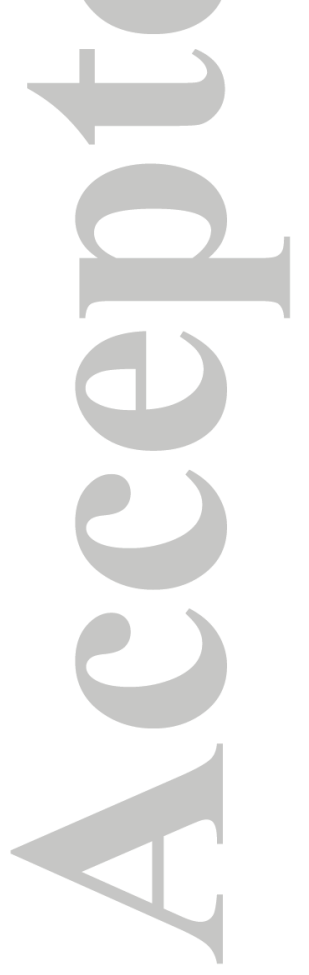




\section{SUPPLEMENTARY MATERIAL}

\section{SUPPLEMENTARY TABLES}

\section{SUPPLEMENTARY TABLE 1: Ran perturbation phenotypes}

Quantification of spindle phenotypes observed in egg extract reactions containing RanT24N or RanQ69L. Data are from 3 independent experiments for each species. Statistical significance was determined by a two-tailed 3x2 Fisher's exact test.

VIDEOS

\section{VIDEO 1: Characterization of $X$. borealis embryo development; $X$. laevis vs. $X$. borealis}

$X$. laevis (left) and $X$. borealis (right) eggs were fertilized with $X$. laevis or $X$. borealis sperm, respectively, and simultaneously imaged in separate dishes. Scale bar corresponds to $200 \mu \mathrm{m}$. The video represents $20 \mathrm{~h}$ played in $15 \mathrm{~s}$ at a rate of 120 frames per second.

\section{SUPPLEMENTARY FIGURE LEGENDS}

\section{SUPPLEMENTARY FIGURE S1: $X$. borealis sperm nuclei length comparison and quantification}

Images of sperm nuclei prepared from $X$. laevis, $X$. tropicalis, and $X$. borealis. DNA signal is shown. Scale bar is $10 \mu \mathrm{m}$. Length $(\mu \mathrm{m})$ is plotted as boxplots for X. laevis (green), X. tropicalis (blue), and $X$. borealis (purple). Each dot represents an individual sperm nucleus ( $\mathrm{n}=31$ for $X$. laevis, $\mathrm{n}=26$ for $X$. tropicalis, $\mathrm{n}=30$ for $X$. borealis), the thicker black line indicates the average, and the outlined gray box represents the standard deviation. Statistical significance was determined by a two-tailed, two-sample unequal variance t-test.

\section{SUPPLEMENTARY FIGURE S2: Analysis of $X$. borealis CSF spindle properties}

(A) Quantification of $X$. borealis CSF spindle dimensions. Length (left), width (middle), and area approximated as an ellipse (right) are plotted $\left(\mu \mathrm{m}\right.$ or $\left.\mu \mathrm{m}^{2}\right)$ as boxplots for $X$. laevis (green), $X$. tropicalis (blue), and $X$. borealis (purple) spindles. Each dot represents an individual spindle 
measurement $(\mathrm{n}=196$ spindles for $X$. laevis, $\mathrm{n}=202$ for $X$. tropicalis, and $\mathrm{n}=304$ for $X$. borealis), the thicker black line indicates the average, and the outlined gray box represents the standard deviation. Statistical significance was determined by a two-tailed, two-sample unequal variance t-test. (B) Microtubule distribution in $X$. laevis (green), X. tropicalis (blue), and $X$. borealis (purple) spindles. Line scans of rhodamine-tubulin signal along the length of the spindle were measured $(\mathrm{n}=243$ spindles for $X$. laevis, $\mathrm{n}=313$ for $X$. tropicalis, and $\mathrm{n}=316$ for $X$. borealis). Spindle length was normalized to $100 \%$ and tubulin intensities were normalized within each dataset. Average tubulin intensities were plotted for each species' spindles. Error bars represent the standard deviation. Representative images of spindles formed from $X$. laevis, $X$. tropicalis, and $X$. borealis sperm nuclei in their respective egg extracts are shown with tubulin signal individually (left) and merged with DNA signal (right). Scale bar is $20 \mu \mathrm{m}$. Data in A-B were reproduced in 3 independent egg extracts for each species. All 3 were used for microtubule distribution analysis in B. Measurements in A were quantified from 2 extracts, processed as squashes for accurate absolute size measurements.

\section{SUPPLEMENTARY FIGURE S3: Effects of cytoplasmic mixing on microtubule distribution}

(A) Microtubule intensity distribution in spindles formed around X. borealis (purple), X. laevis (green), and $X$. tropicalis (blue) sperm nuclei in $X$. borealis egg extract $(\mathrm{n}=316$ for $X$. borealis sperm nuclei, $\mathrm{n}=307$ spindles for $X$. laevis sperm nuclei, and $\mathrm{n}=307$ for $X$. tropicalis sperm nuclei, from 3 independent $X$. borealis egg extracts). (B) Microtubule distribution in spindles formed in mixed extracts of $X$. laevis (green) and $X$. borealis (purple) ( $\mathrm{n}=301$ for $100 \% X$. borealis, $0 \%$ X. laevis; $\mathrm{n}=338$ for $75 \%$ X. borealis, $25 \%$ X. laevis; $\mathrm{n}=322$ for $50 \% X$. borealis, $50 \% X$. laevis; $\mathrm{n}=295$ for $25 \% X$. borealis, $75 \% X$. laevis; and $\mathrm{n}=240$ for $0 \% X$. borealis, $100 \% X$. laevis over 3 independent experiments). (C) Microtubule distribution in spindles formed in mixed extracts of $X$. tropicalis (blue) and $X$. borealis (purple) ( $\mathrm{n}=283$ for $100 \% X$. borealis, $0 \% X$. tropicalis; $\mathrm{n}=272$ for $75 \% X$. borealis, $25 \% X$. tropicalis; $\mathrm{n}=260$ for $50 \% X$. borealis, $50 \% X$. tropicalis; $\mathrm{n}=272$ for $25 \%$ X. borealis, $75 \%$ X. tropicalis; and $\mathrm{n}=305$ for $0 \%$ $X$. borealis, $100 \% X$. tropicalis over 3 independent experiments). In A-C, line scans of rhodamine-tubulin signal along the length of the spindle were measured. Spindle length was 
normalized to $100 \%$ and tubulin intensities were normalized within each dataset. Average tubulin intensities were plotted. Error bars represent the standard deviation.

\section{SUPPLEMENTARY FIGURE S4: Localization of XMAP215 on X. borealis spindles}

(A) XMAP215 localization in X. laevis (green), X. tropicalis (blue), and X. borealis (purple) spindles. Line scans of Alexa Fluor 488 signal along the length of the spindle were measured (n $=420$ spindles for $X$. laevis, $\mathrm{n}=390$ for $X$. tropicalis, and $\mathrm{n}=338$ for $X$. borealis, from 3 independent egg extracts for each species). Spindle length was normalized to $100 \%$, and XMAP215 intensities were normalized within each dataset. Average XMAP215 intensities were plotted for each species' spindles. Error bars represent the standard deviation. Representative images of spindles formed around $X$. laevis, $X$. tropicalis, and $X$. borealis sperm nuclei in their respective egg extracts are shown with XMAP215 signal in gray scale (left) and merged with tubulin and DNA signals (right). Scale bar is $20 \mu \mathrm{m}$. (B) Western blot of X. laevis, X. tropicalis, and $X$. borealis extracts probed for XMAP215. XMAP215 band integrated density is plotted as columns for $X$. laevis (green), X. tropicalis (blue), and X. borealis (purple) extracts, with 3 independent extracts analyzed for each species. Band intensity was normalized to the integrated density of the corresponding Ran loading control. Error bars represent the standard deviation. Statistical significance was determined by a two-tailed, two-sample unequal variance t-test.

\section{SUPPLEMENTARY FIGURE S5: Protein sequence alignments of Xenopus katanin}

Protein sequences of $X$. tropicalis, $X$. laevis, and $X$. borealis katanin were aligned using Clustal Omega (default parameters). The $X$. borealis sequence is $97.6 \%$ identical to that of $X$. tropicalis, and $97.0 \%$ identical to $X$. laevis. The red box indicates the key regulatory serine residue at position 131.

\section{SUPPLEMENTARY FIGURE S6: Protein sequence alignments of Xenopus TPX2}

Protein sequences of $X$. tropicalis, $X$. laevis, and $X$. borealis TPX2 were aligned using Clustal Omega (default parameters). The $X$. borealis sequence is $75.7 \%$ identical to $X$. tropicalis, and $85.8 \%$ and $91.3 \%$ identical to sequences from the two subgenomes of $X$. laevis, $\mathrm{L}$ and $\mathrm{S}$, respectively. The red box indicates the key 7 amino acids at position 619-624 that are absent from $X$. tropicalis and regulate microtubule nucleation activity. 
Figure 1
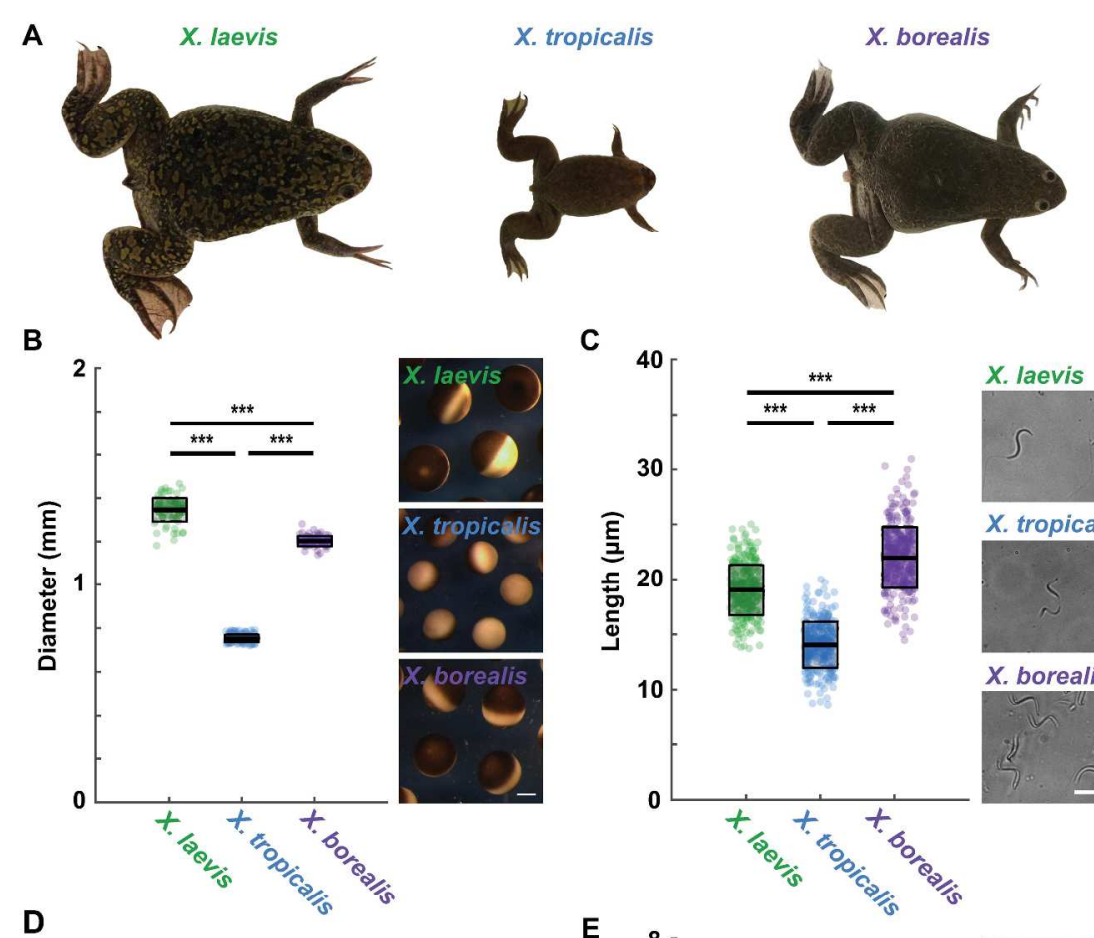

C
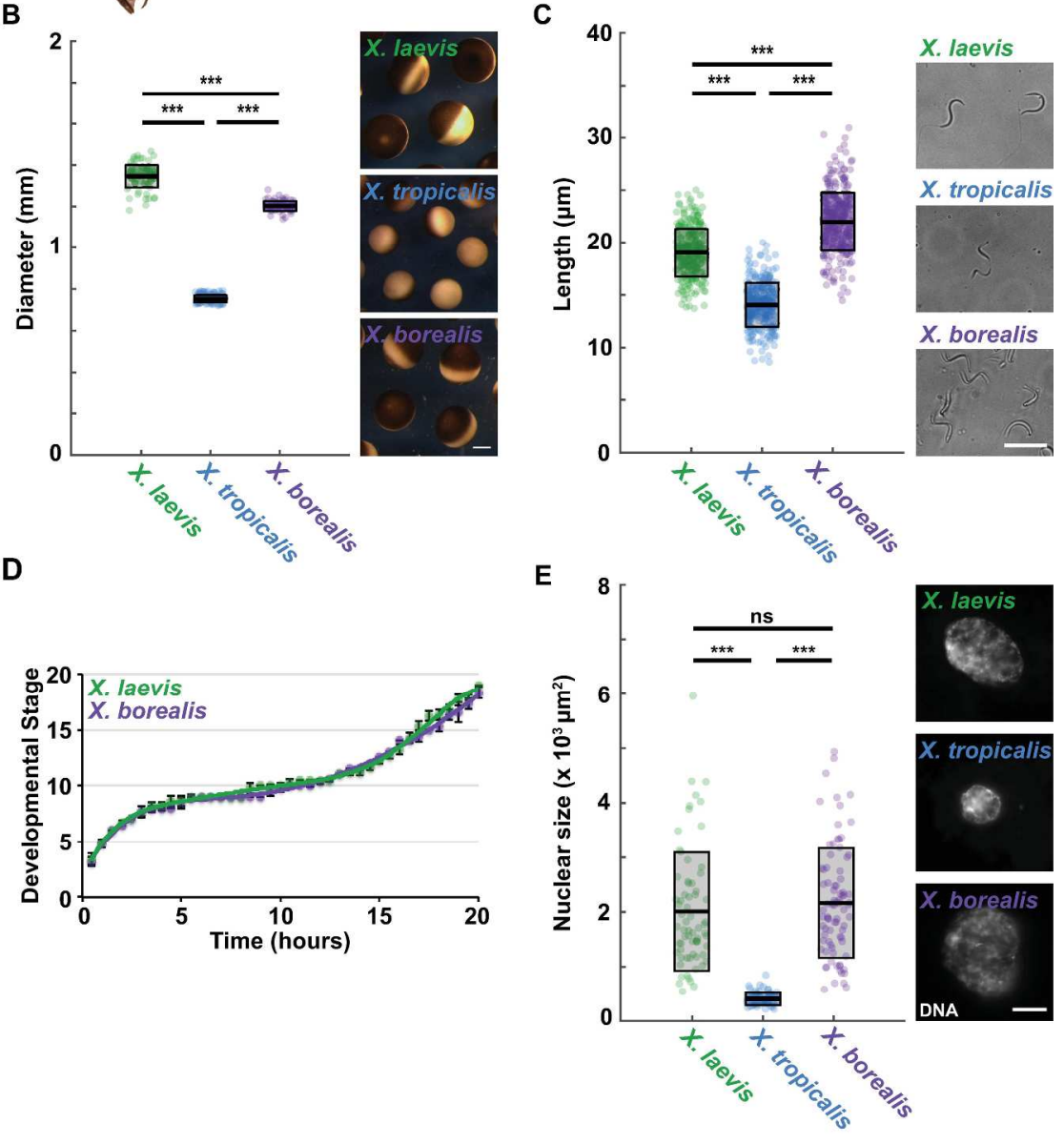

Figure 1

$269 \times 362 \mathrm{~mm}(300 \times 300$ DPI $)$ 
Figure 2

A

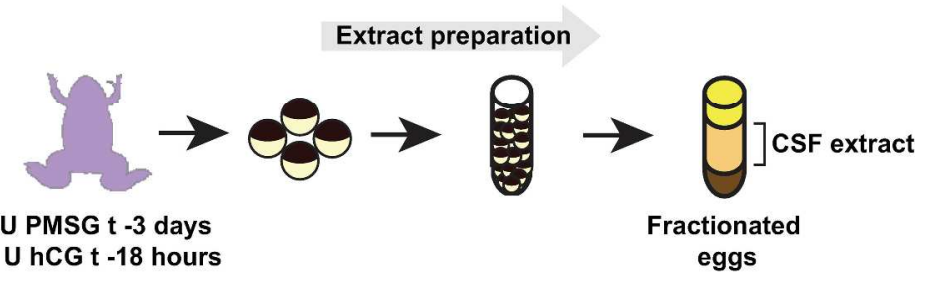
$300 \mathrm{U}$ hCG $\mathrm{t}-18$ hours

Spindle reactions
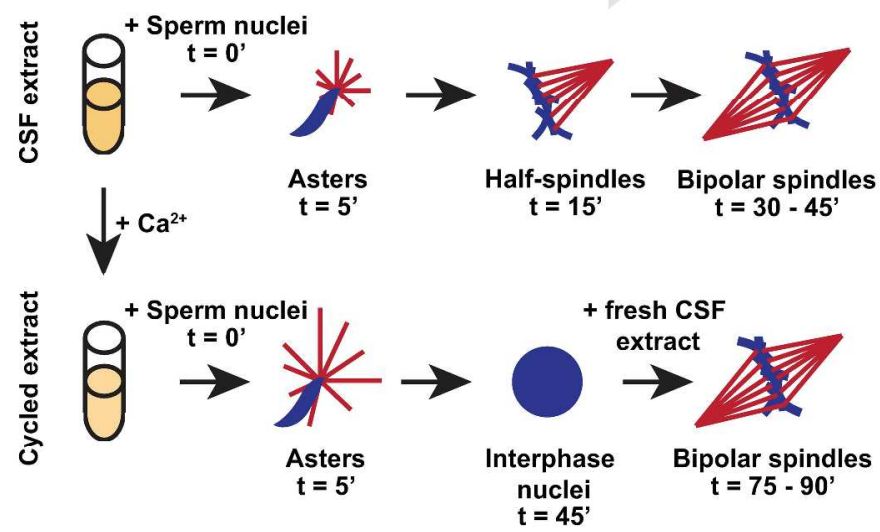

B
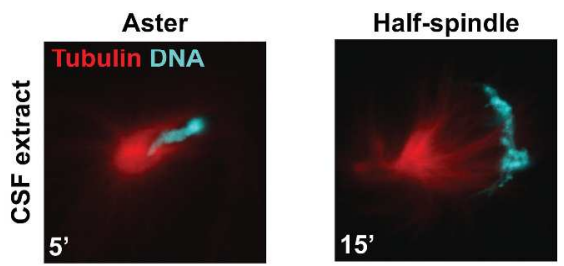

Bipolar spindle

C

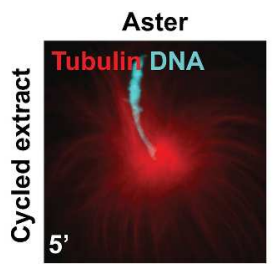

Interphase nucleus
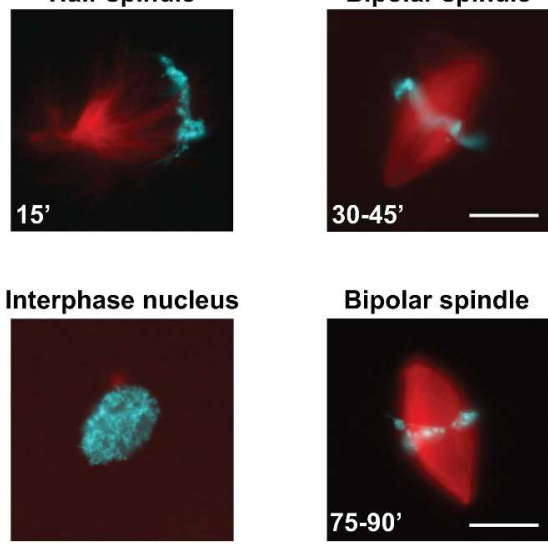

Figure 2

$256 \times 384 \mathrm{~mm}(300 \times 300$ DPI $)$ 
Figure 3

A
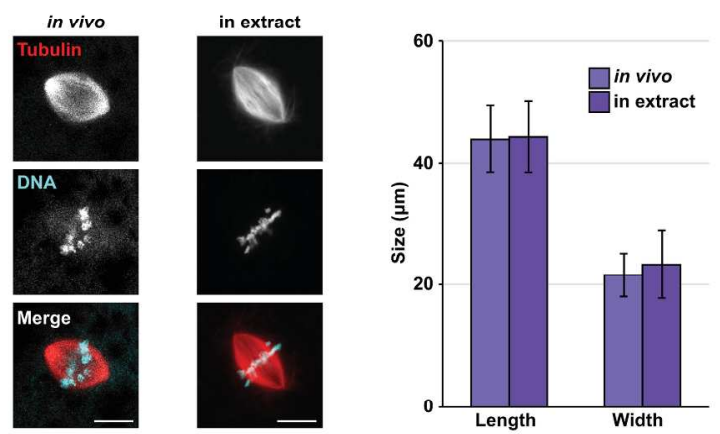

B
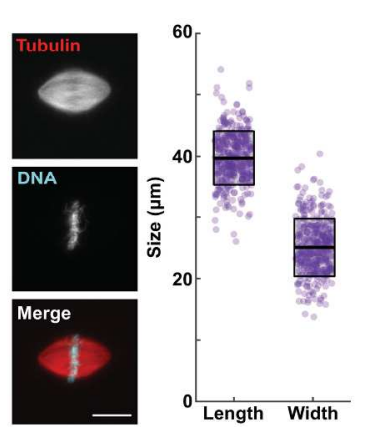

C

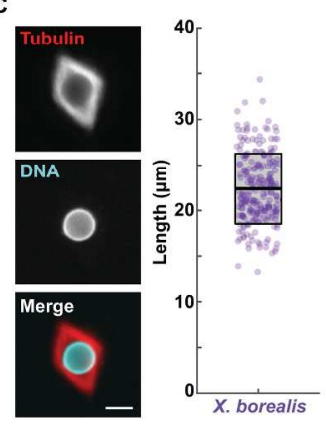

Figure 3

$231 \times 178 \mathrm{~mm}(300 \times 300 \mathrm{DPI})$

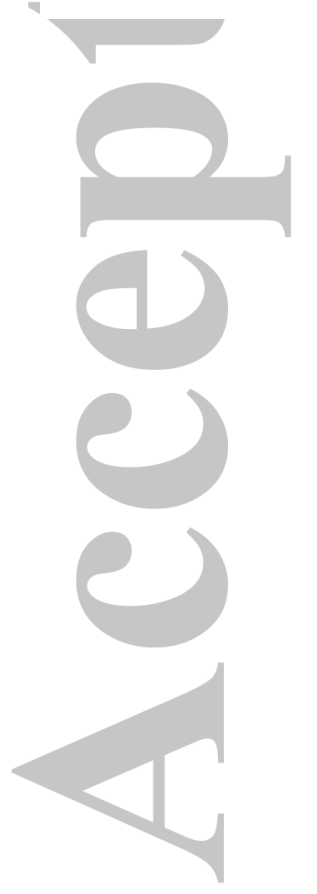




\section{Figure 4}
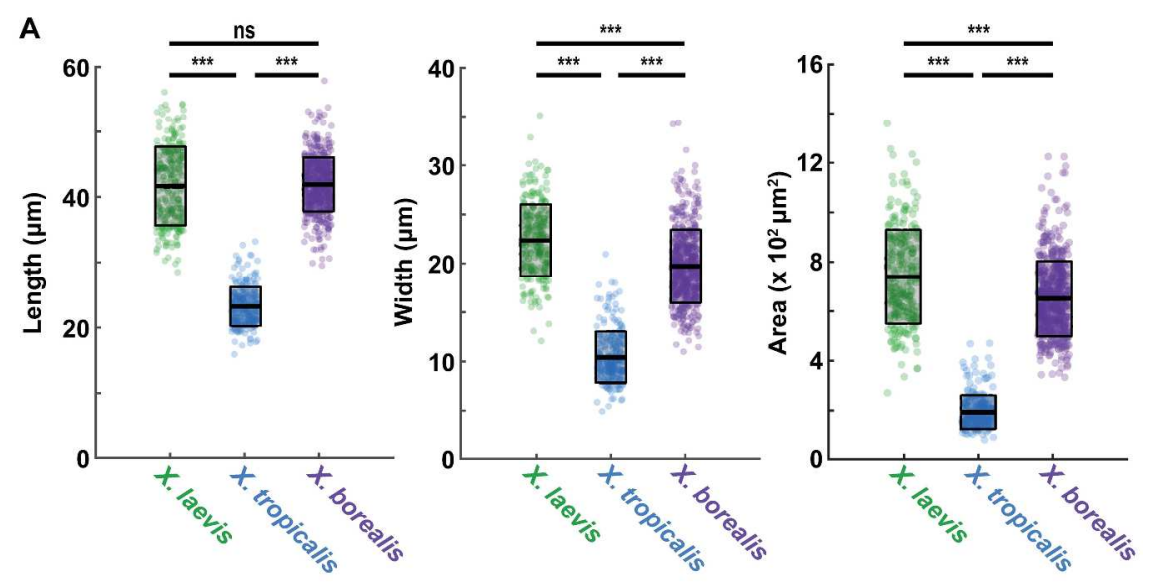

B

X. laevis
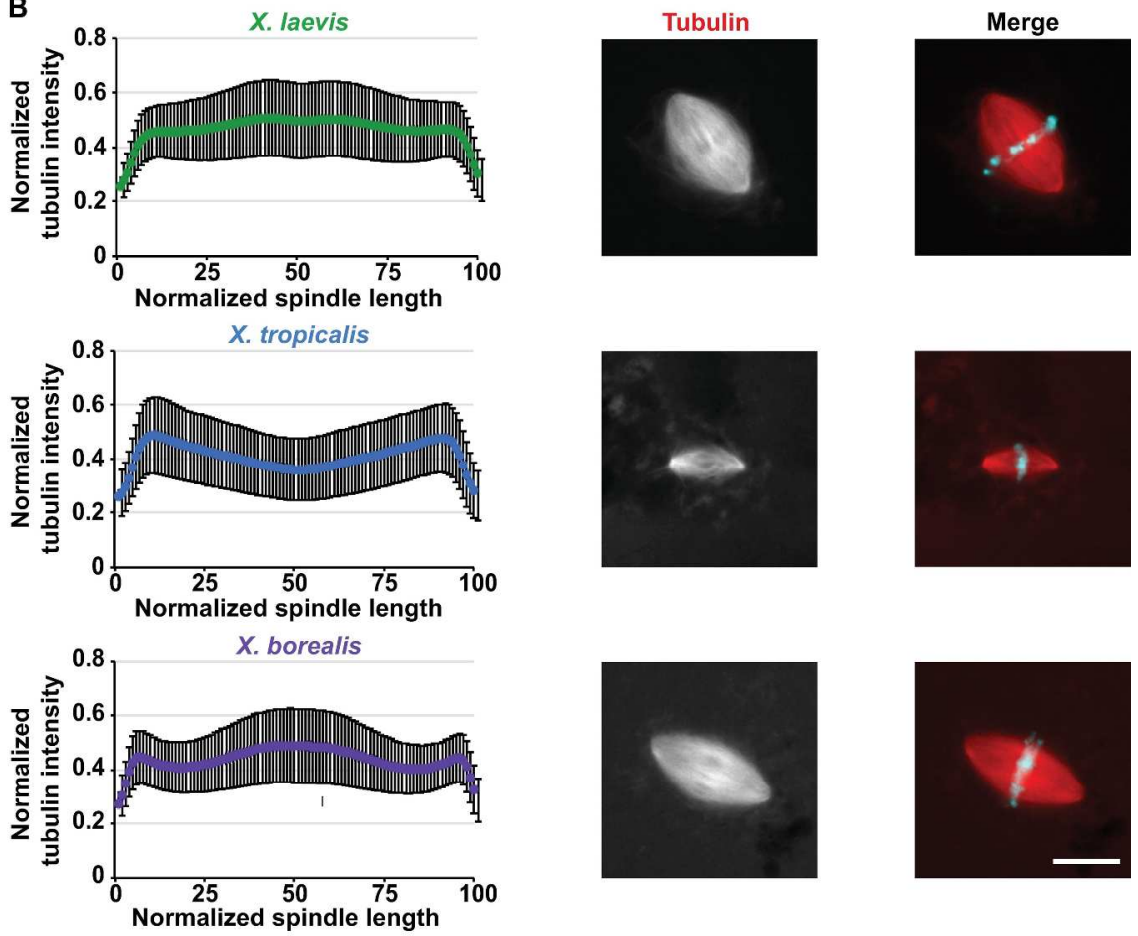

Figure 4

$266 \times 360 \mathrm{~mm}(300 \times 300$ DPI $)$ 
Figure 5

A
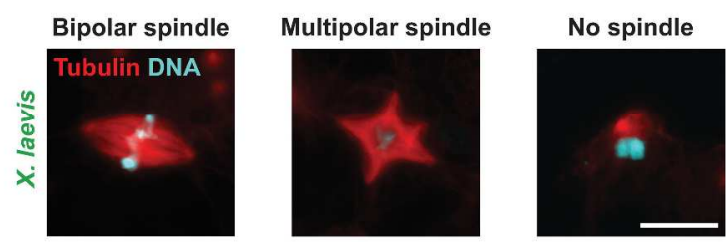

B

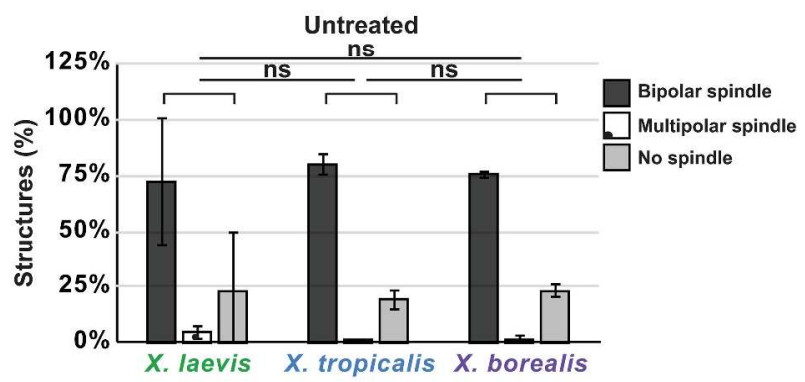

C

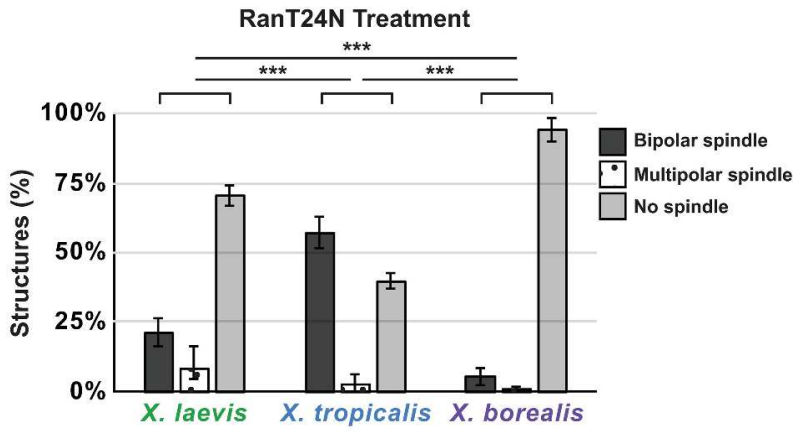

D

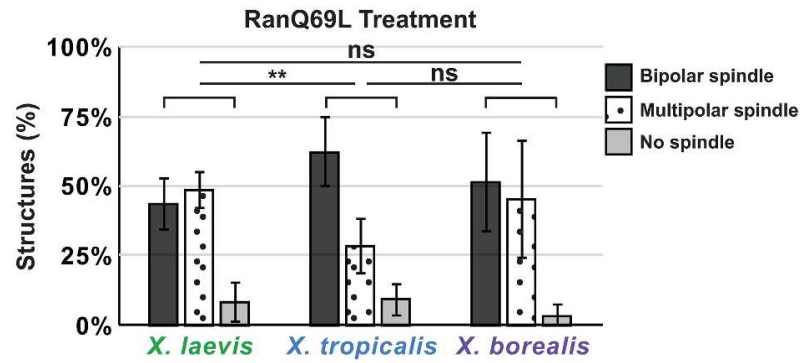

Figure 5

$267 \times 471 \mathrm{~mm}(300 \times 300$ DPI $)$ 
Figure 6

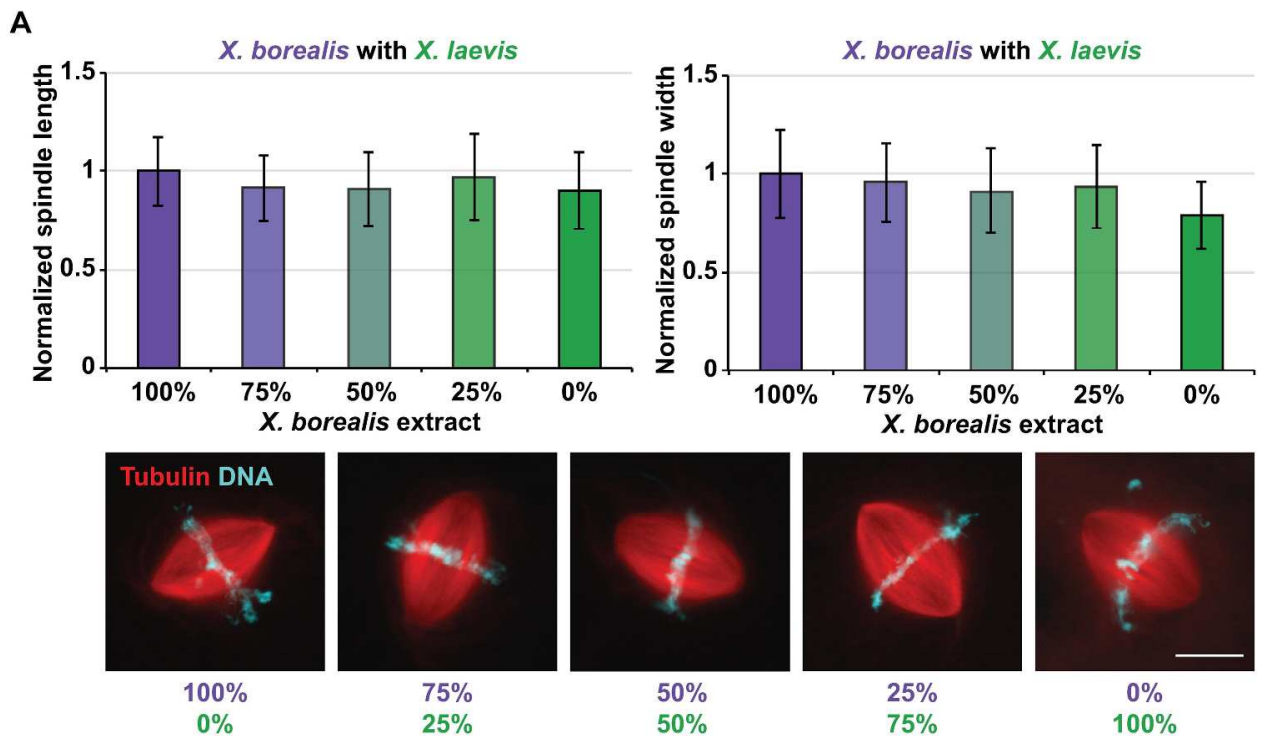

B

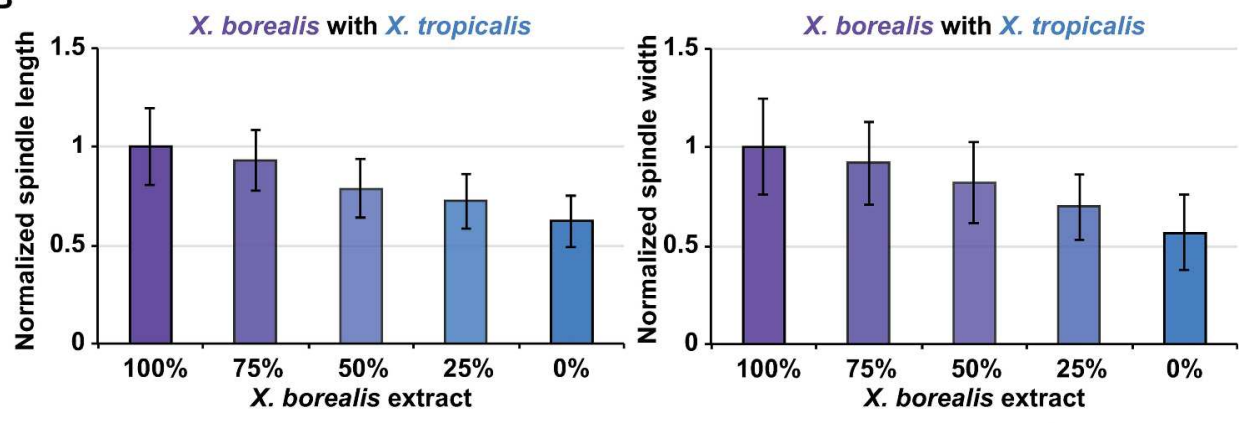

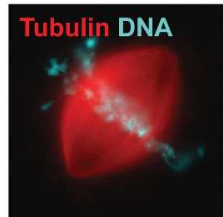

$100 \%$

$0 \%$

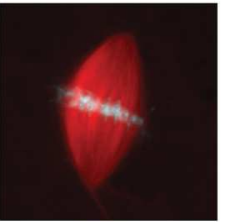

$75 \%$

$25 \%$

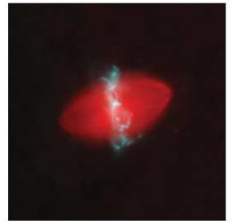

$50 \%$

$50 \%$

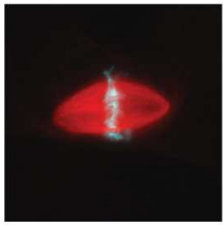

$25 \%$

$75 \%$

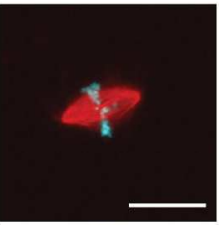

$0 \%$

Figure 6

$256 \times 320 \mathrm{~mm}(300 \times 300$ DPI $)$ 
Figure 7

A

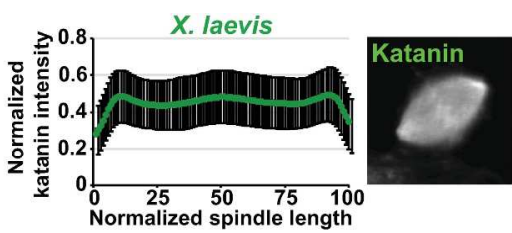

N.tropicalis

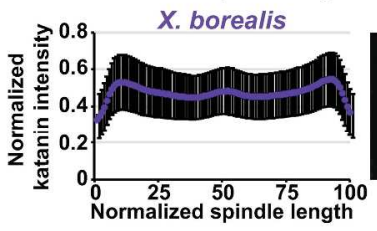

C

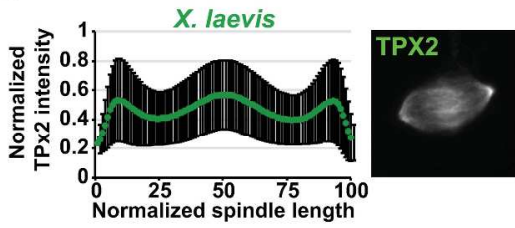

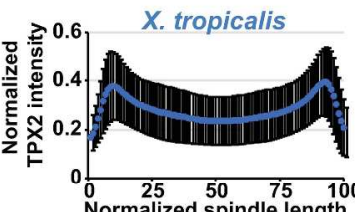

$X$ borealis

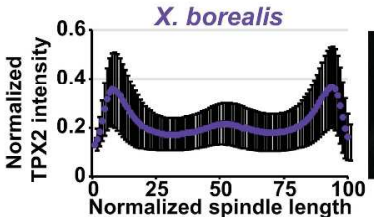

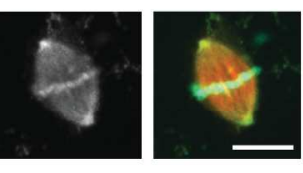
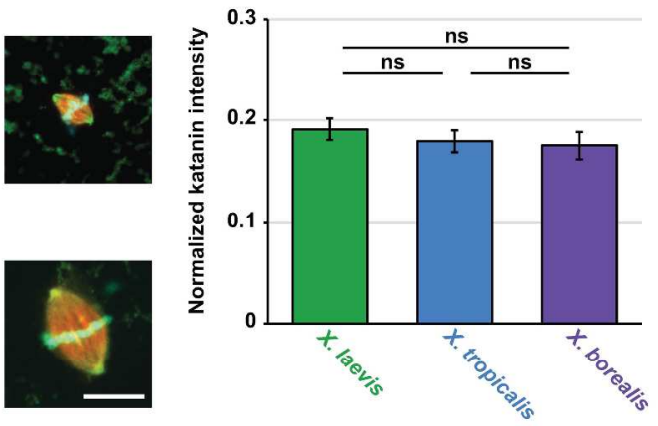

D
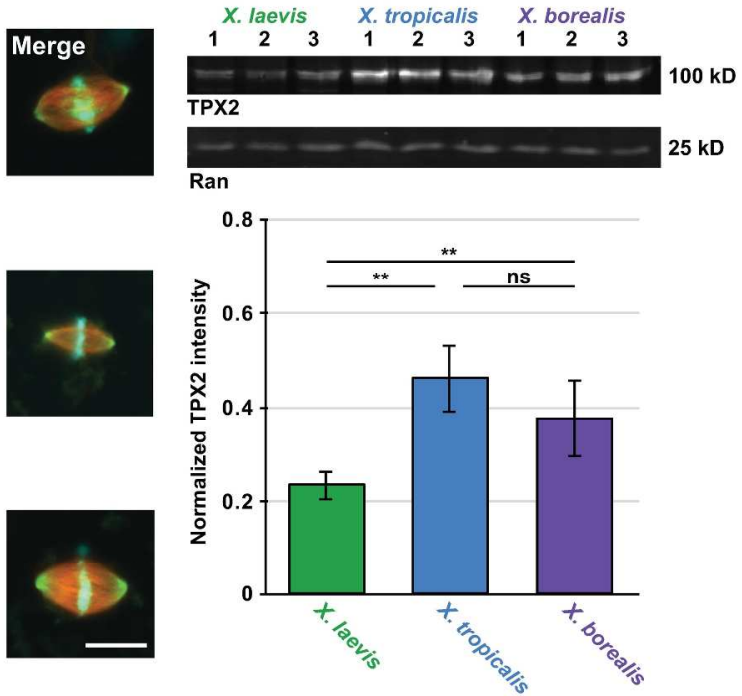

Figure 7

$259 \times 317 \mathrm{~mm}(300 \times 300 \mathrm{DPI})$ 


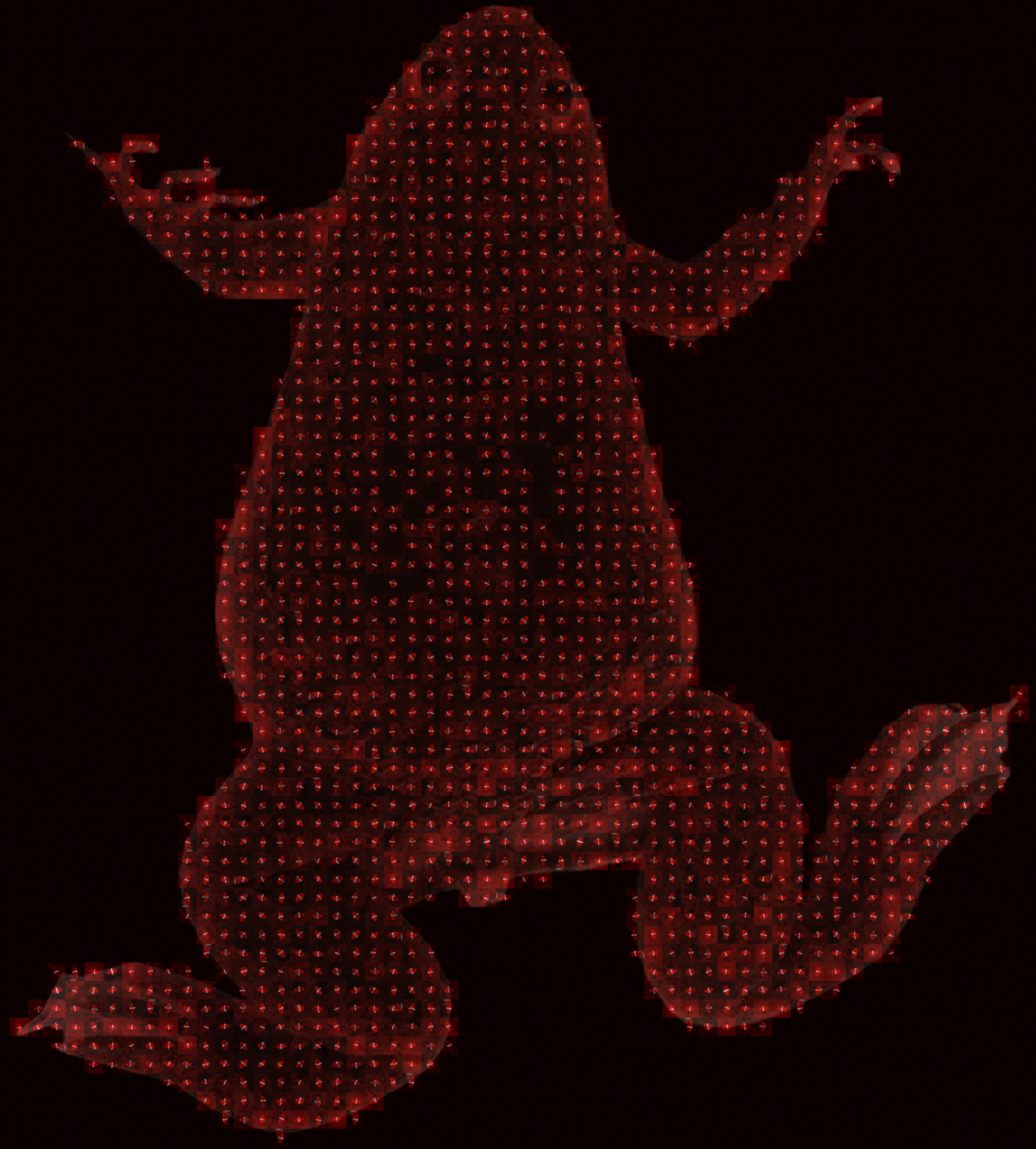

